\title{
On the Whitham hierarchy: dressing scheme, string equations and additional symmetries
}

\author{
Manuel Mañas \\ Departamento de Física Teórica II, Universidad Complutense \\ 28040-Madrid, Spain \\ email: manuel.manas@fis.ucm.es \\ Elena Medina \\ Departamento de Matemáticas, Universidad de Cádiz \\ 11520-Puerto Real, Cádiz, Spain \\ email: elena.medina@uca.es \\ Luis Martínez Alonso \\ Departamento de Física Teórica II, Universidad Complutense \\ 28040-Madrid, Spain \\ email: luism@fis.ucm.es
}

1991 MSC: 58B20

\begin{abstract}
A new description of the universal Whitham hierarchy in terms of a factorization problem in the Lie group of canonical transformations is provided. This scheme allows us to give a natural description of dressing transformations, string equations and additional symmetries for the Whitham hierarchy. We show how to dress any given solution and prove that any solution of the hierarchy may be undressed, and therefore comes from a factorization of a canonical transformation. A particulary important function, related to the $\tau$-function, appears as a potential of the hierarchy. We introduce a class of string equations which extends and contains previous classes of string equations considered by Krichever and by Takasaki and Takebe. The scheme is also applied for an convenient derivation of additional symmetries. Moreover, new functional symmetries of the Zakharov extension of the Benney gas equations are given and the action of additional symmetries over the potential in terms of linear PDEs is characterized.
\end{abstract}

\section{Introduction}

Dispersionless integrable models, see [14],[31], [32] and [11], appear in the analysis of various problems in physics and applied mathematics from the theory of quantum fields, see [12] and 
[2], to the theory of conformal and quasiconformal maps on the complex plane, see [5]-[10]. The new millennium brought new applications of these models, see [29]-[28], in different areas, as for example integrable deformations of conformal maps e interfacial processes.

The Krichever's universal Whitham hierarchies, see [11]-[12], are the integrable systems involved in these applications. These hierarchies include as particular cases the dispersionless KP, dispersionless modified KP and dispersionless Toda hierarchies, see [29]-[1] and [8]-[23]. The role of twistor or string equations for studying dispersionless integrable models was emphasized by Takasaki and Takebe in [23]-[27]. Solutions of these string equations have attractive mathematical properties as well as interesting physical meaning.

The objective of this paper is to formulate the factorization problem for the zero genus Whitham hierarchy within the context of Lie groups of symplectic transformations, and to give a natural and general formalism for string equations and additional symmetries. In particular, we characterize an special class of string equations, related to a Virasoro algebra. It turns out that this class determines not only the solutions of the algebraic orbits of the Whitham hierarchy [12] but also the solutions arising in the above mentioned applications of dispersionless integrable models [18]-[19].

The layout of the paper is as follows. In $\S 2$ we introduce the Lie algebraic splitting for Hamiltonian vector fields and the corresponding factorization problem for canonical transformations. Then, in $\S 3$ we show how deformations of the factorization problem of canonical transformations lead to solutions of the Whitham hierarchy. We remark a particular system of equations within the hierarchy: The Boyer-Finley-Benney equations, which extend the Boyer-Finley and the Benney equations, respectively. Here we also introduce a potential function of the hierarchy from which all the fields of the hierarchy are gotten by appropriate derivations. In a forthcoming paper [20] we show that this function is the $x$-derivative of $-\log \tau$, where $\tau$ is the $\tau$-function of the hierarchy. We proof that any solution of the Whitham hierarchy may be obtained from a factorization problem; i.e. it may be undressed. To conclude the section we extend the factorization scheme to get the dressing of any given solution of the Whitham hierarchy. In section 4 we consider the string equations in the context of the factorization problem. For that aim we introduce the Orlov-Schulman functions, and show that the factorization problem leads to string equations. Thus all solutions of the Whitham hierarchy fulfill certain set of string equations. In [20] we show that any solution of the string equations is a solution of the Whitham hierarchy. We finish this section by introducing some particular factorization problems and the corresponding string equations which generalize and contains as particular cases, the string equations of Krichever and of Takasaki-Takebe. Finally, $\S 5$ is devoted to the study of additional symmetries of the Whitham hierarchy. First we derive the additional symmetries from the factorization problem, and then characterize its action over the potential function of the hierarchy. We compute the additional symmetries of the mentioned Boyer-Finley-Benney system and obtain a set of explicit functional symmetries. In particular, for the Zakharov extension of the Benney system we get explicit functional symmetries depending on three arbitrary functions of variable. We conclude by considering the action of Virasoro type of additional symmetries on our extension of the Krichever and Takasaki-Takebe string equations and showing that solutions of string equations are invariant solutions under a Lie algebra of additional symmetries, which contains two set of Virasoro algebras. 


\section{The factorization problem}

\subsection{Lie algebraic setting}

We present a splitting which is inspired in [22] and in [15], where it was used for a better understanding of harmonic maps and chiral models. The factorization problem technique was applied to the dispersionless KP hierarchy in [7], and is inspired in the dressing method proposed by Takasi and Takabe in the series of papers [23]-[27].

Given the set $\left\{q_{\mu}^{(0)}\right\}_{\mu=0}^{M} \subset \overline{\mathbb{C}}, q_{0}^{(0)}=\infty$, of punctures in the extended complex plane, we introduce the local parameters $p_{\mu}^{-1}$ where

$$
p_{\mu}= \begin{cases}p, & \mu=0 \\ \left(p-q_{i}^{(0)}\right)^{-1}, & \mu=i \in \mathbb{S}\end{cases}
$$

and

$$
\mathbb{S}:=\{1, \ldots, M\} .
$$

For each set of punctures we consider the set $\mathcal{R}$ of rational functions in $p$ with poles at the punctures; i.e., the functions $f=f(p)$ of the form

$$
f:=\sum_{\mu=0}^{M} \sum_{n=0}^{N_{\mu}} a_{n}^{\mu} p_{\mu}^{n},
$$

where $N_{\mu} \in \mathbb{N}$. In this paper we use Greek letters like $\mu$ to denote an index that runs from 0 to $M$, and Italic letters like $i$, when it runs from 1 to $M$.

For each puncture $q_{\mu}^{(0)}$ we consider the set $\mathcal{L}_{\mu}=\mathbb{C}\left(p_{\mu}\right)$ of formal Laurent series in $p_{\mu}$ and the subset $\mathcal{L}_{\mu}^{-}$defined as

$$
\mathcal{L}_{\mu}^{-}:= \begin{cases}p^{-1} \mathbb{C}\left[\left[p^{-1}\right]\right], & \text { for } \mu=0, \\ \mathbb{C}\left[\left[p-q_{i}^{(0)}\right]\right], & \text { for } \mu=i \in \mathbb{S} .\end{cases}
$$

Here $\mathbb{C}[[p]]$ denotes the set of formal power series in $p$. Finally, we define

$$
\mathcal{L}:=\bigoplus_{\mu=0}^{M} \mathcal{L}_{\mu}, \quad \mathcal{L}^{-}:=\bigoplus_{\mu=0}^{M} \mathcal{L}_{\mu}^{-} .
$$

Given an element $\left(f_{0}, f_{1}, \ldots, f_{M}\right) \in \mathcal{L}$, let $f_{(\mu,+)}$ be the polynomial in $p_{\mu}^{-1}$ such that $\tilde{f}_{\mu}^{-}:=$ $f_{\mu}-f_{(\mu,+)} \in \mathcal{L}_{\mu}^{-}$. Then, there exists a unique rational function $f \in \mathcal{R}$ whose principal parts at $q_{\mu}^{(0)}$ are given by $f_{(\mu,+)}$ (observe the normalization condition at $\infty$ ), namely

$$
f=\sum_{\mu=0}^{M} f_{(\mu,+)} .
$$

Moreover, we have a unique splitting of $f_{\mu}$ of the form

$$
f_{\mu}=f_{\mu}^{-}+f
$$

with

$$
f_{\mu}^{-}:=\tilde{f}_{\mu}^{-}-\sum_{\nu \neq \mu} \tilde{f}_{\nu}^{-} \in \mathcal{L}_{\mu}^{-}
$$




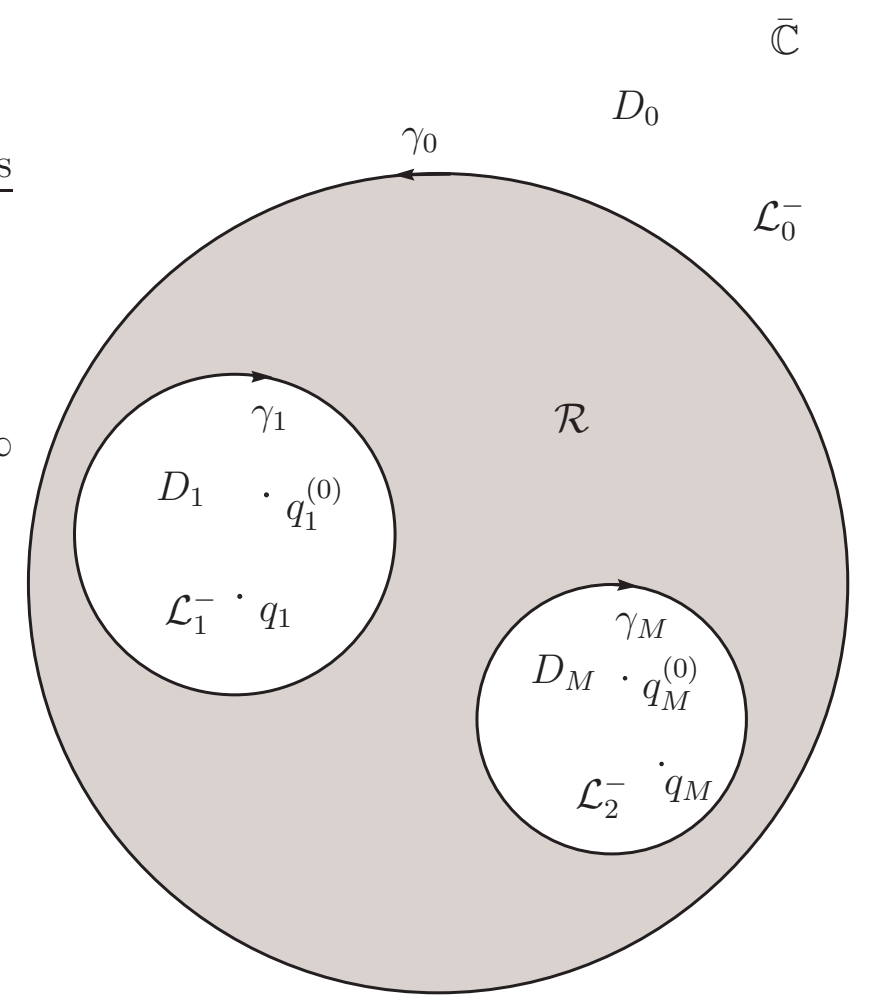

Figure 1: Graphical illustration of the splitting.

Therefore, we conclude that the following splitting

$$
\mathcal{L}=\mathcal{L}^{-} \oplus \mathcal{R}
$$

holds.

The above construction can be extended in the following manner. Let us consider, for $i \in \mathbb{S}$, the disk $D_{i}$ containing the point $q_{i}^{(0)}$ with border the clockwise oriented circle $\gamma_{i}:=\partial D_{i}$, and also the disk $D_{0}$, centered at 0 , which contains all the other disks $D_{i}, i=1, \ldots, M$, with border the counter-clockwise oriented circle $\gamma_{0}:=\partial D_{0}$. Let $D:=D_{0}^{c} \cup\left(\bigcup_{i=1}^{M} D_{i}\right)$ and $\gamma:=\bigcup_{\mu=0}^{M} \gamma_{\mu}$, here $D_{0}^{c}:=\overline{\mathbb{C}} \backslash D_{0}$ is the complementary set of the disk $D_{0}$. We will consider the completion of $\mathcal{L}$ as the set of complex functions over $\Gamma$. We complete the rational splitting by extending $\mathcal{L}_{\mu}^{-}$as those complex functions over $\gamma_{\mu}$ which admit analytic extensions to its interior, and for $\mu=0$ such that the extension vanishes at $\infty$. Then, $\mathcal{L}^{-}=\oplus_{\mu=0}^{M} \mathcal{L}_{\mu}^{-}$and $\mathcal{R}$ is the set of complex functions on $\Gamma$ such that they do have an holomorphic extension to $\mathbb{C} \backslash D$. In this context (1) also holds. We refer to Figure 1 for a graphical illustration of the rational splitting and its completion. Now, we shall extend the above splitting to the Lie algebra of symplectic vector fields. In spite that normally the coordinates $(p, x)$ are real, here we will consider that they take complex values. This extension does not affect the standard local symplectic constructions.

The local Hamiltonian vector fields

$$
\boldsymbol{X}=A(p, x) \frac{\partial}{\partial p}+B(p, x) \frac{\partial}{\partial x},
$$

are the divergence free vector fields $A_{p}+B_{x}=0$, and locally there exists a Hamiltonian function 
$H$ such that

$$
A=-\frac{\partial H}{\partial x}, \quad B=\frac{\partial H}{\partial p} .
$$

The Poisson bracket in the set $\mathcal{F}$ of differentiable functions of $p$ and $x$ is locally given by

$$
\{H, \tilde{H}\}=\frac{\partial H}{\partial p} \frac{\partial \tilde{H}}{\partial x}-\frac{\partial H}{\partial x} \frac{\partial \tilde{H}}{\partial p},
$$

and the pair $\mathfrak{g}:=(\mathcal{F},\{\cdot, \cdot\})$ is a Lie algebra. The set of inner derivations of $\mathfrak{g}$

$$
\text { ad } H:=\{H, \cdot\}=\frac{\partial H}{\partial p} \frac{\partial}{\partial x}-\frac{\partial H}{\partial x} \frac{\partial}{\partial p}=\boldsymbol{X}_{H}
$$

may be locally identified with the set of Hamiltonian vector fields. If fact, the set of locally Hamiltonian vector fields constitute a Lie algebra under the Lie bracket given by the Lie derivative of vector fields, and we have that

$$
\left[\boldsymbol{X}_{H}, \boldsymbol{X}_{\tilde{H}}\right]=\boldsymbol{X}_{\{H, \tilde{H}\}},
$$

so that the mapping $H \rightarrow \boldsymbol{X}_{H}$ is a Lie algebra homomorphism with kernel given by the constant functions; i.e., the center of the Lie algebra of Hamiltonian functions.

We denote by $\mathfrak{g}_{\mu}, \mathfrak{g}_{\mu}^{-}$, and $\mathfrak{r}$, the Lie subalgebras of $\mathfrak{g}$ such that the corresponding Lie algebras of Hamiltonian vector fields ad $\mathfrak{g}_{\mu}$, ad $\mathfrak{g}_{\mu}^{-}$and ad $\mathfrak{r}$ are built up from vector fields with coefficients in $\mathcal{L}_{\mu}, \mathcal{L}_{\mu}^{-}$and $\mathcal{R}$, respectively. Here, we suppose that the coefficients of the vector fields are complex valued functions. Let us describe in more detail these Lie algebras:

1. The Lie algebra $\mathfrak{r}$. The components $A$ and $B$ of a Hamiltonian vector field

$$
\operatorname{ad} H=A \frac{\partial}{\partial p}+B \frac{\partial}{\partial x} \in \operatorname{ad} \mathfrak{r}
$$

are $A=-H_{x}$ and $B=H_{p}$ with

$$
H=\sum_{n=0}^{N_{0}} h_{n}(x) p^{n}+\sum_{i=1}^{M}\left(h_{i 0} \log \left(p-q_{i}^{(0)}\right)+\sum_{j=1}^{N_{i}} \frac{h_{i j}(x)}{\left(p-q_{i}^{(0)}\right)^{j}}\right),
$$

and $h_{i 0, x}=0$.

2. The Lie algebras $\mathfrak{g}_{\mu}$. The components $A_{\mu}$ and $B_{\mu}$ of a Hamiltonian vector field

$$
\text { ad } H_{\mu}=A_{\mu} \frac{\partial}{\partial p}+B_{\mu} \frac{\partial}{\partial x} \in \operatorname{ad} \mathfrak{g}_{\mu}
$$

are $A_{\mu}=-H_{\mu, x}$ and $B_{\mu}=H_{\mu, p}$ with

$$
H_{\mu}=h_{\mu 0} \log \left(p_{\mu}\right)+\sum_{n \gg-\infty} h_{\mu n}(x) p_{\mu}^{-n}, \quad h_{\mu 0, x}=0 .
$$

3. The Lie algebra $\mathfrak{g}^{-}$. The components $A_{\mu}$ and $B_{\nu}$ of a vector field

$$
\text { ad } H_{\mu}=A_{\mu} \frac{\partial}{\partial p}+B_{\mu} \frac{\partial}{\partial x} \in \mathfrak{g}_{\mu}^{-},
$$

are $A_{\mu}=-H_{\mu, x}$ and $B_{\mu}=H_{\mu, p}$ with

$$
H_{0}=h_{00} \log p+\sum_{n=1}^{\infty} h_{0 n}(x) p^{-n}, \quad H_{i}=\sum_{n=0}^{\infty} h_{i n}(x)\left(p-q_{i}^{(0)}\right)^{n},
$$

with $h_{00, x}=0$. 
Now, we define the Lie algebras

$$
\mathfrak{g}:=\bigoplus_{\mu=0}^{M} \mathfrak{g}_{\mu}, \quad \mathfrak{g}^{-}:=\bigoplus_{\mu=0}^{M} \mathfrak{g}_{\mu}^{-},
$$

and realize that, modulo constants, the splitting (1) in this context is

$$
\operatorname{ad} \mathfrak{g}=\operatorname{ad} \mathfrak{g}^{-} \dot{+} \operatorname{ad} \mathfrak{r}
$$

which in turn is equivalent to

$$
\mathfrak{g}=\mathfrak{g}^{-} \dot{+} \mathfrak{r} .
$$

The Lie algebras $\mathfrak{g}_{i}$, for $i=1, \ldots, M$, have a further splitting into three Lie subalgebras:

$$
\mathfrak{g}_{i}^{-}=\mathfrak{g}_{i}^{0} \dot{+} \mathfrak{g}_{i}^{1} \dot{+} \mathfrak{g}_{i}^{>},
$$

where

$$
\mathfrak{g}_{i}^{0}:=\left\{h_{i 0}(x)\right\}, \quad \mathfrak{g}_{i}^{1}=\left\{h_{i 1}(x)\left(p-q_{i}^{(0)}\right)\right\}, \quad \mathfrak{g}_{i}^{>}:=\left\{h_{i 2}(x)\left(p-q_{i}^{(0)}\right)^{2}+h_{i 3}(x)\left(p-q_{i}^{(0)}\right)^{3}+\cdots\right\}
$$

and $\left\{\mathfrak{g}_{i}^{0} \dot{+} \mathfrak{g}_{i}^{1}, \mathfrak{g}_{i}^{>}\right\} \subset \mathfrak{g}_{i}^{>}$. The above splitting induces the following splitting into Lie subalgebras of divergence free vector fields

$$
\operatorname{ad} \mathfrak{g}_{i}^{-}=\operatorname{ad} \mathfrak{g}_{i}^{0} \dot{+} \operatorname{ad} \mathfrak{g}_{i}^{1} \dot{+} \operatorname{ad} \mathfrak{g}_{i}^{>} .
$$

\subsection{Lie group setting}

We now extend the previous construction from the context of Lie algebras to the corresponding Lie groups of canonical transformations. Associated with each Hamiltonian vector field $\boldsymbol{X}_{H}=$ ad $H$ we have the corresponding Hamilton's equations $\dot{p}=-H_{x}, \dot{x}=H_{p}$ that when integrated provides us with a flow $\Phi_{t}^{H}$, a 1-parameter group of symplectic diffeomorphims, $(p(t), x(t))=$ $\Phi_{t}^{H}\left(p_{0}, x_{0}\right)$ for given initial conditions $\left.(p, x)\right|_{t=0}=\left(p_{0}, x_{0}\right)$. The exponential mapping is just the evaluation at $t=1$; i.e. $\exp \boldsymbol{X}_{H}=\Phi_{t=1}^{H}$. The group of symplectic diffeomorphims is a smooth regular Lie group with Lie algebra given by the set of Hamiltonian vector fields [9]. Symplectic diffeormorphims are also known as canonical transformations.

It can be shown [9] that the adjoint action of the group of symplectic diffeomorphims on its Lie algebra (i.e., the set of Hamiltonian vector fields) is given by the action of the corresponding induced flow:

$$
\operatorname{Ad} \exp \left(s \boldsymbol{X}_{H}\right)\left(\boldsymbol{X}_{\tilde{H}}\right)=\left(\Phi_{-s}^{H}\right)^{*} \boldsymbol{X}_{\tilde{H}}=T \Phi_{s}^{H} \circ \boldsymbol{X}_{\tilde{H}} \circ \Phi_{-s}^{H}=\boldsymbol{X}_{\left(\Phi_{-s}^{H}\right) * \tilde{H}}=\boldsymbol{X}_{\operatorname{Ad} \exp (s H) \tilde{H}},
$$

where

$$
\operatorname{Ad} \exp (s H) \tilde{H}:=\left(\Phi_{-s}^{H}\right)^{*} \tilde{H}=\mathrm{e}^{s \operatorname{ad} H} \tilde{H}=\sum_{l=0}^{\infty} \frac{(s \operatorname{ad} H)^{l}}{l !} \tilde{H} .
$$

That is, modulo constants, the adjoint action of a symplectic diffeomorphism of the form $\exp \left(\boldsymbol{X}_{H}\right)$ acts on the Hamiltonian functions as $\mathrm{e}^{\text {ad } H}$ :

$$
\exp \left(\boldsymbol{X}_{H}\right) \stackrel{\operatorname{Ad}}{\longrightarrow} \mathrm{e}^{\text {ad } H}
$$


The rational splitting of Lie algebras of Hamiltonian vector fields may be exponentiated to a Birkhoff type factorization problem

$$
\exp \left(\boldsymbol{X}_{\mu}\right)=\exp \left(\boldsymbol{X}_{\mu}^{-}\right)^{-1} \circ \exp (\boldsymbol{X}) \text { with } \boldsymbol{X}_{\mu} \in \operatorname{ad} \mathfrak{g}_{\mu}, \boldsymbol{X}_{\mu}^{-} \in \operatorname{ad} \mathfrak{g}_{\mu}^{-} \text {and } \boldsymbol{X} \in \operatorname{ad} \mathfrak{r}
$$

where we are now dealing with complex vector fields.

We will consider a particular class of Hamiltonians, namely those of the following form

$$
T_{\mu}:=\left(1-\delta_{\mu 0}\right) t_{\mu 0} \log p_{\mu}+\sum_{n=1+\delta_{\mu 0}}^{\infty} t_{\mu n} p_{\mu}^{n} .
$$

Given initial canonical transformations $\Phi_{\mu}, \mu=0, \ldots, M$, we consider deformations $\exp \left(\boldsymbol{X}_{T_{\mu}}\right) \circ$ $\Phi_{\mu}$ which area new canonical transformations that now depend on the deformation or time parameters

$$
\boldsymbol{t}:=\left(t_{\mu n}\right)
$$

We will consider the factorization

$$
\exp \left(\boldsymbol{X}_{T_{\mu}}\right) \circ \Phi_{\mu}=\exp \left(\boldsymbol{X}_{\mu}^{-}\right)^{-1} \circ \exp (\boldsymbol{X}) \text { with } \boldsymbol{X}_{\mu}^{-} \in \operatorname{ad} \mathfrak{g}_{\mu}^{-} \text {and } \boldsymbol{X} \in \operatorname{ad} \mathfrak{r}
$$

Equation (4) is fulfilled if the following factorization problem is satisfied

$$
\mathrm{e}^{\operatorname{ad} T_{\mu}} \mathrm{e}^{\operatorname{ad} G_{\mu}}=\mathrm{e}^{-\operatorname{ad} H_{\mu}^{-}} \mathrm{e}^{\text {ad } H} \text {, with } H_{\mu}^{-} \in \mathfrak{g}_{\mu}^{-} \text {and } H \in \mathfrak{r},
$$

where,

$$
\Phi_{\mu}=\exp \boldsymbol{X}_{G_{\mu}}, \boldsymbol{X}_{\mu}^{-}=\boldsymbol{X}_{H_{\mu}^{-}}, \boldsymbol{X}=\boldsymbol{X}_{H} .
$$

The existence problem for (5) will not be treated here. Anyhow, we will assume that all times $\left|t_{\mu n}\right|$ and initial conditions are small enough to ensure that such factorization exists (notice the trivial existence for $T_{\mu}=0$ and $G_{\mu}=0$ ).

Observe that given a set of initial conditions $\left\{G_{\mu}\right\}_{\mu=0}^{M}$ the factorization problem (5) consists in finding $H_{\mu}^{-}, \mu=0, \ldots, M$, and $H$ as functions of $\boldsymbol{t}$. Let us right-multiply both terms of the equality (5) by a term of the form $\mathrm{e}^{\text {ad } G}$, where $G \in \mathfrak{r}$. On the left-hand term we have $\mathrm{e}^{\text {ad } T_{\mu}} \mathrm{e}^{\text {ad } \tilde{G}_{\mu}}$, where the new initial conditions are

$$
\tilde{G}_{\mu}:=C\left(G_{\mu}, G\right):=G_{\mu}+G+\frac{1}{2}\left\{G_{\mu}, G\right\}+\frac{1}{12}\left(\left\{G_{\mu},\left\{G_{\mu}, G\right\}+\left\{G,\left\{G, G_{\mu}\right\}\right\}\right)+\cdots,\right.
$$

and $C(\cdot, \cdot)$ is the Campbell-Hausdorff series in Dynkin form, so that

$$
\mathrm{e}^{\operatorname{ad} G_{\mu}} \mathrm{e}^{\operatorname{ad} G}=\mathrm{e}^{\operatorname{ad} \tilde{G}_{\mu}} .
$$

A solution of this new factorization problem is given by $\tilde{H}_{\mu}^{-}=H_{\mu}^{-}$and $\tilde{H}=C(H, G) \in \mathfrak{r}$, so that $H_{\mu}^{-}$remains invariant. Let us now left-multiply both terms of the equality by $\mathrm{e}^{\text {ad } c_{\mu}^{-}(p)}$, with $c_{\mu}^{-} \in \mathfrak{c}_{\mu}^{-}$, being $\mathfrak{c}_{\mu}^{-} \subset \mathfrak{g}_{\mu}^{-}$the Abelian subalgebra of Hamiltonians in $\mathfrak{g}_{\mu}^{-}$which only depend on $p$. As $\left\{c_{\mu}^{-}, T_{\mu}\right\}=0$ we have $\mathrm{e}^{\operatorname{ad} c_{\mu}^{-}} \mathrm{e}^{\operatorname{ad} T_{\mu}} \mathrm{e}^{\operatorname{ad} G_{\mu}}=\mathrm{e}^{\operatorname{ad} T_{\mu}} \mathrm{e}^{\text {ad } \tilde{G}_{\mu}}$ with $\tilde{G}_{\mu}:=C\left(c_{\mu}^{-}, G_{\mu}\right)$. The solution of the transformed factorization problem (5) is given by $\tilde{H}_{\mu}^{-}=C\left(c_{\mu}^{-}, H_{\mu}^{-}\right)$and $\tilde{H}=H$.

Therefore, once we have a solution $\left(H_{\mu}^{-}, H\right)$ for an initial condition $G_{\mu}$ it is trivial to find solutions $\left(\tilde{H}_{\mu}^{-}, \tilde{H}\right)$ for initial conditions $C\left(\mathfrak{c}_{\mu}^{-}, C\left(G_{\mu}, \mathfrak{r}\right)\right)$. The orbits $\mathrm{e}^{\text {ad } \mathfrak{c}_{\mu}^{-}} \mathrm{e}^{\text {ad } G_{\mu}} \mathrm{e}^{\text {ad } \mathfrak{r}}$ describe the moduli space of solutions to the factorization problem (5). Thus, if we concentrate on the 
right action of $\mathfrak{r}$, we may take $G_{\mu} \in \mathfrak{g}_{\mu}^{-}$and the right coset $\mathrm{e}^{\text {ad } G_{\mu}} \mathrm{e}^{\text {ad } \mathfrak{r}}$ (or the Hamiltonian $\left.C\left(G_{\mu}, \mathfrak{r}\right)\right)$ as the point in the moduli.

As we will see the factorization problem (5) for the action of symplectic diffeomorphims on the set of functions (observables) implies the Whitham hierarchy. Therefore, the factorization problem (4) for symplectic diffeormorphism is also associated with the Whitham hierarchy. To get these results we will use a well known tool in the theory of regular Lie groups: the right logarithmic derivative as defined in [9], see the Appendix. If we have a smooth curve $H: \mathcal{T} \rightarrow C^{\infty}(\mathcal{N})$, assuming that $\mathcal{T}$ is the time manifold with local coordinates given by $\boldsymbol{t}=\left(t_{\mu n}\right)$ and denoting $\partial_{\mu m}:=\frac{\partial}{\partial t_{\mu n}}$, the right logarithmic derivative is

$$
\delta \exp \left(\boldsymbol{X}_{H}\right)\left(\partial_{\mu n}\right)=\int_{0}^{1}\left(\Phi_{-s}^{H}\right)^{*}\left(T_{\boldsymbol{t}} \boldsymbol{X}_{H}\left(\partial_{\mu n}\right)\right) \mathrm{d} s=\boldsymbol{X}_{\delta \mathrm{e}^{\mathrm{ad} H}\left(\partial_{\mu n}\right)},
$$

where

$$
\delta \mathrm{e}^{\operatorname{ad} H}\left(\partial_{\mu n}\right):=\int_{0}^{1}\left(\Phi_{-s}^{H}\right)^{*}\left(\partial_{\mu n} H\right) \mathrm{d} s=\sum_{l=0}^{\infty} \frac{(\operatorname{ad} H)^{l}}{(l+1) !} \partial_{\mu n} \tilde{H} .
$$

In particular,

$$
\delta \exp \left(\boldsymbol{X}_{T_{\mu}}\right)\left(\partial_{\mu n}\right)=\boldsymbol{X}_{\frac{\partial T_{\mu}(p)}{\partial t \mu n}} \text { with } \frac{\partial T_{\mu}(p)}{\partial t_{\mu n}}= \begin{cases}p_{\mu}^{n}, & n \neq 0 \\ \log \left(p-q_{i}^{(0)}\right), & n=0, \mu=i .\end{cases}
$$

Now, we are ready to take right logarithmic derivatives, using (95), of the factorization problem (4),

$$
\delta \exp \left(\boldsymbol{X}_{\nu}^{-}\right)\left(\partial_{\mu n}\right)+\delta_{\mu \nu} \operatorname{Ad} \exp \left(\boldsymbol{X}_{\mu}^{-}\right)\left(\boldsymbol{X}_{\frac{\partial T_{\mu}(p)}{\partial t_{\mu n}}}\right)=\delta \exp (\boldsymbol{X})\left(\partial_{\mu n}\right),
$$

Which, using the corresponding Hamiltonian generators

$$
\boldsymbol{X}_{\mu}^{-}=\boldsymbol{X}_{H_{\mu}^{-}}, \quad \boldsymbol{X}=\boldsymbol{X}_{H}
$$

we get, modulo constants, the following system

$$
\delta \mathrm{e}^{\operatorname{ad} H_{\nu}^{-}}\left(\partial_{\mu n}\right)+\delta_{\mu \nu} \mathrm{e}^{\operatorname{ad} H_{\mu}^{-}}\left(\frac{\partial T_{\mu}(p)}{\partial t_{\mu n}}\right)=\delta \mathrm{e}^{H}\left(\partial_{\mu n}\right),
$$

which may be derived directly from (5) by taking right logarithmic derivatives.

\section{Dressing methods for the Whitham hierarchy}

In this section we analyze how the factorization problem (5) is related with the Whitham hierarchy and its dressing transformations. We first show that (5) leads to the Whitham hierarchy, defining the Lax functions a zero-curvature forms. Then, we construct a potential function $h_{01}$ of this hierarchy, and as we shall show in the forthcoming paper [20] $h_{01}=-(\log \tau)_{x}$ in terms of the $\tau$-function of the hierarchy. We also proof that any solution of the Whitham hierarchy is related to a factorization problem, via an undressing procedure. Finally, we show how the factorization problem scheme can be extended to generate dressing transformations of the Whitham hierarchy. 


\subsection{From the factorization problem to the Whitham hierarchy}

We are now ready to proof that (5) is described differentially by the Whitham hierarchy

Theorem 1. Given a solution of the factorization problem (5),

$$
\mathrm{e}^{\operatorname{ad} T_{\mu}} \mathrm{e}^{\operatorname{ad} G_{\mu}}=\mathrm{e}^{-\operatorname{ad} H_{\mu}^{-}} \mathrm{e}^{\operatorname{ad} H}, \quad H_{\mu}^{-} \in \mathfrak{g}_{\mu}^{-}, H \in \mathfrak{r},
$$

then:

1. The Lax functions

$$
z_{\mu}:=\mathrm{e}^{\mathrm{ad} H_{\mu}^{-}} p_{\mu}
$$

are of the form

$$
z_{\mu}= \begin{cases}p+\sum_{l=1}^{\infty} d_{0 l} p^{-l}, & \mu=0, \\ \frac{d_{i-1}}{p-q_{i}}+\sum_{l=0}^{\infty} d_{i l}\left(p-q_{i}\right)^{l}, & \mu=i \in \mathbb{S}\end{cases}
$$

for some functions $q_{i}$ and $d_{\mu m}$ defined in terms of the coefficients of $H_{\mu}^{-}$.

2. The functions

$$
\Omega_{\mu n}:= \begin{cases}\left(z_{\mu}^{n}\right)_{(\mu,+)}, & n>\delta_{\mu 0}, \\ -\log \left(p-q_{i}\right), & n=0, \quad \mu=i \in \mathbb{S}\end{cases}
$$

where $(\cdot)_{(i,+)}$ projects in the span $\left\{\log \left(p-q_{i}\right),\left(p-q_{i}\right)^{-l}\right\}_{l=1}^{\infty}$ and $(\cdot)_{(0,+)}$ onto the span of $\left\{p^{l}\right\}_{l=0}^{\infty}$, satisfy the zero-curvature equations

$$
\frac{\partial \Omega_{\mu n}}{\partial t_{\nu l}}-\frac{\partial \Omega_{\nu l}}{\partial t_{\mu n}}+\left\{\Omega_{\mu n}, \Omega_{\nu l}\right\}=0
$$

moreover

$$
\Omega_{\mu n}=\delta \mathrm{e}^{\mathrm{ad} H}\left(\partial_{\mu n}\right) .
$$

3. The Lax functions $z_{\mu}$ are subject to the Whitham hierarchy:

$$
\frac{\partial z_{\nu}}{\partial t_{\mu n}}=\left\{\Omega_{\mu n}, z_{\nu}\right\}
$$

Proof. We now proceed to show that (9) implies the Whitham hierarchy. In the analysis of (9) is convenient to distinguish between the cases $\mu=i \neq 0$ and $\mu=0$.

1. The case $\mu=i \in \mathbb{S}$.

We factor $\mathrm{e}^{\text {ad } H_{i}^{-}}=\mathrm{e}^{\text {ad } H_{i 0}} \mathrm{e}^{\text {ad } H_{i 1}} \mathrm{e}^{\text {ad } H_{i>}>}$, with

$H_{i 0}=h_{i 0}(x), \quad H_{i 1}=h_{i 1}(x)\left(p-q_{i}^{(0)}\right), \quad H_{i>}=h_{i 2}(x)\left(p-q_{i}^{(0)}\right)^{2}+h_{i 3}(x)\left(p-q_{i}^{(0)}\right)^{3}+\cdots$.

Now, we study the cases $m>0$ and $m=0$ : 
(a) $m>0$

We get

$$
\delta \mathrm{e}^{\operatorname{ad} H_{i 0}}\left(\partial_{i n}\right)+\mathrm{e}^{\operatorname{ad} H_{i 0}}\left(\delta \mathrm{e}^{\operatorname{ad} H_{i 1}}\left(\partial_{i n}\right)+\mathrm{e}^{\operatorname{ad} H_{i 1}}\left(\delta \mathrm{e}^{\operatorname{ad} H_{i>}}\left(\partial_{i n}\right)\right)\right)+z_{i}^{n}=\delta \mathrm{e}^{\operatorname{ad} H}\left(\partial_{i n}\right)
$$

It can be proved that

$$
\begin{aligned}
\delta \mathrm{e}^{\operatorname{ad} H_{i 0}}\left(\partial_{i n}\right) & =\partial_{i n} h_{i 0}, \\
\delta \mathrm{e}^{\operatorname{ad} H_{i 1}}\left(\partial_{i n}\right) & =\frac{\partial_{i n} X_{i}}{X_{i, x}}\left(p-X_{i, x} q_{i}^{(0)}\right), \quad \text { with } \int_{x}^{X_{i}} \frac{\mathrm{d} x}{h_{i 1}(x)}=1, \\
\delta \mathrm{e}^{\operatorname{ad} H_{i>}>}\left(\partial_{i n}\right) & =\partial_{i n} h_{i 2}\left(p-q_{i}^{(0)}\right)^{2}+\left(\partial_{i n} h_{i 3}+h_{i 2} \partial_{i n} h_{i 2, x}-h_{i 2, x} \partial_{i n} h_{i 2}\right)\left(p-q_{i}^{(0)}\right)^{3}+\cdots, \\
\mathrm{e}^{\operatorname{ad} H_{i 0}}\left(f(x)\left(p-q_{i}^{(0)}\right)^{n}\right) & =f(x)\left(p-q_{i}^{(0)}-h_{i 0, x}\right)^{n}, \\
\mathrm{e}^{\operatorname{ad} H_{i 1}}\left(f(x)\left(p-q_{i}^{(0)}\right)^{n}\right) & =\frac{\left.f\right|_{x=X_{i}}\left(p-X_{i, x} q_{i}^{(0)}\right)^{n}, \text { and in particular } \mathrm{e}^{\text {ad } H_{i 1}} x=X_{i}}{\left(X_{i, x}\right)^{n}} \\
\mathrm{e}^{\operatorname{ad} H_{i>}}\left(\frac{1}{\left(p-q_{i}^{(0)}\right)^{n}}\right) & =\left(\frac{1}{p-q_{i}^{(0)}}+h_{i 2, x}+\left(h_{i 3, x}+h_{i 2} h_{i 2, x x}\right)\left(p-q_{i}^{(0)}\right)+\cdots\right)^{n} .
\end{aligned}
$$

Therefore, defining

$$
q_{i}:=X_{i, x} q_{i}^{(0)}+h_{i 0, x},
$$

we deduce that (15) can be written as

$$
\partial_{i n} h_{i 0}+\frac{\partial_{i n} X_{i}}{X_{i, x}}\left(p-q_{i}\right)+\frac{\left.\left(\partial_{i n} h_{i 2}\right)\right|_{x=X_{i}}}{\left(X_{i, x}\right)^{2}}\left(p-q_{i}\right)^{2}+\cdots+z_{i}^{n}=\delta \mathrm{e}^{\text {ad } H}\left(\partial_{i n}\right)
$$

with

$$
z_{i}:=\mathrm{e}^{\mathrm{ad} H_{i}^{-}}\left(\frac{1}{p-q_{i}^{(0)}}\right)=\frac{d_{i-1}}{p-q_{i}}+\sum_{l=0}^{\infty} d_{i l}\left(p-q_{i}\right)^{l},
$$

where, for example,

$$
d_{i-1}:=X_{i, x}, \quad d_{i 0}:=\left.h_{i 2, x}\right|_{x=X_{i}}, \quad d_{i 1}:=\frac{\left.\left(h_{i 3, x}+h_{i 2} h_{i 2, x x}\right)\right|_{x=X_{i}}}{X_{i, x}}
$$

We have assumed that $T_{\mu}$ and $G_{\mu}$ are small enough to ensure that the function $q_{i}=X_{i, x} q_{i}^{(0)}+h_{i 0, x}$ belongs to the interior of $U_{q_{i}^{(0)}}$ (so that $X_{i, x} \approx 1$ and $h_{i 0, x} \approx 0$ ). Thus, (16) implies

$$
\mathfrak{r} \ni \delta \mathrm{e}^{\text {ad } H}\left(\partial_{i m}\right)=\left(z_{i}^{m}\right)_{(i,+)}=: \Omega_{i m} .
$$

For example,

$$
\Omega_{i 1}=\frac{d_{i-1}}{p-q_{i}}, \quad \Omega_{i 2}=\frac{d_{i-1}^{2}}{\left(p-q_{i}\right)^{2}}+\frac{2 d_{i-1} d_{i 0}}{p-q_{i}} .
$$

(b) $m=0$

In this case we have

$$
\partial_{i 0} h_{i 0}+\frac{\partial_{i 0} X_{i}}{X_{i, x}}\left(p-q_{i}\right)+\frac{\left.\left(\partial_{i 0} h_{i 2}\right)\right|_{x=X_{i}}}{\left(X_{i, x}\right)^{2}}\left(p-q_{i}\right)^{2}+\cdots+\log z_{i}=\delta \mathrm{e}^{\text {ad } H}\left(\partial_{i 0}\right) .
$$


Notice that

$\log z_{i}:=-\log \left(p-q_{i}\right)+\log \left(X_{i, x}+\left.h_{i 2, x}\right|_{x=X_{i}}\left(p-q_{i}\right)+\frac{\left.\left(h_{i 3, x}+h_{i 2} h_{i 2, x}\right)\right|_{x=X_{i}}}{X_{i, x}}\left(p-q_{i}\right)^{2}+\cdots\right)$,

and hence

$$
\mathfrak{r} \ni \delta \mathrm{e}^{\operatorname{ad} H}\left(\partial_{i, 0}\right)=\left(\log z_{i}\right)_{(i,+)}=: \Omega_{i 0}
$$

with

$$
\Omega_{i 0}=-\log \left(p-q_{i}\right)
$$

2. $\mu=0$

In this case we have

$$
\delta \mathrm{e}^{\operatorname{ad} H_{0}^{-}}\left(\partial_{0 n}\right)+z_{0}^{n}=\delta \mathrm{e}^{\operatorname{ad} H}\left(\partial_{0 n}\right) .
$$

with

$$
\mathrm{e}^{\operatorname{ad} H_{0}^{-}}=\mathrm{e}^{\operatorname{ad} H_{0>}} \mathrm{e}^{\operatorname{ad}\left(t_{00} \log p\right)}, \quad H_{0>}=h_{01} p^{-1}+h_{02} p^{-2}+\cdots,
$$

where $t_{00}$, which is not a time parameter, does not depend on $x$. Notice that

$$
z_{0}=\mathrm{e}^{\operatorname{ad} H_{0}^{-}}(p)=p+\sum_{l=1}^{\infty} d_{0 l} p^{-l}
$$

where, for example,

$$
d_{01}:=-h_{01, x}, \quad d_{02}:=-h_{02, x} .
$$

An analysis of equation (18) allows us to write

$$
\mathfrak{r} \ni \delta \mathrm{e}^{\operatorname{ad} H}\left(\partial_{0 n}\right)=\left(z_{0}^{n}\right)_{(0,+)}=: \Omega_{0 n},
$$

for example

$$
\Omega_{02}=p^{2}+2 d_{01} .
$$

From

$$
\Omega_{\mu n}=\delta \mathrm{e}^{\operatorname{ad} H}\left(\partial_{\mu n}\right)
$$

and (96) we deduce the zero-curvature conditions (13).

3. From (97) we have

$$
\frac{\partial z_{\nu}}{\partial t_{\mu n}}=\left\{\delta \mathrm{e}^{\mathrm{ad} H_{\nu}^{-}}\left(\partial_{\mu n}\right), z_{\nu}\right\}
$$

that recalling (9) reads

$$
\frac{\partial z_{\nu}}{\partial t_{\mu n}}=\left\{\delta \mathrm{e}^{\operatorname{ad} H}\left(\partial_{\mu n}\right), z_{\nu}\right\}
$$

and we deduce (14).

As a byproduct of the above proof we have the following 
Proposition 1. Given solutions $H_{\mu}^{-}$and $H$ of the factorization problem (5) such that

$$
\mathrm{e}^{\operatorname{ad} H_{\mu}^{-}}= \begin{cases}\mathrm{e}^{\operatorname{ad}\left(\sum_{l=1}^{\infty} h_{0 l}(x) p^{-l}\right)} \mathrm{e}^{\operatorname{ad}\left(t_{00} \log p\right)}, & \mu=0, \\ \mathrm{e}^{\operatorname{ad} h_{i 0}(x)} \mathrm{e}^{\operatorname{ad} h_{i 1}(x)\left(p-q_{i}^{(0)}\right)} \mathrm{e}^{\operatorname{ad}\left(\sum_{l=2}^{\infty} h_{i l}(x)\left(p-q_{i}^{(0)}\right)^{l}\right)}, & \mu=i \in \mathbb{S},\end{cases}
$$

then

$$
t_{00}=-\sum_{i=1}^{M} t_{i 0}
$$

and the coefficients of the Lax functions satisfy

$$
\begin{aligned}
& q_{i}=X_{i, x} q_{i}^{(0)}+h_{i 0, x}, \quad \int_{x}^{X_{i}} \frac{\mathrm{d} x}{h_{i 1}(x)}=1, \\
& d_{i-1}=X_{i, x} \\
& d_{i l}=\left.\left(h_{i l+2, x}+f_{i l}\left(h_{i l+1}, \ldots, h_{i 2}\right)\right)\right|_{x=X_{i}} X_{i, x}^{-l}, \quad l \geq 0 \\
& d_{0 l}=-h_{0 l, x}+f_{0 l}\left(h_{0 l-1}, \ldots, h_{01}\right) \text {, }
\end{aligned}
$$

where $f_{\mu l}$ are differential polynomials.

Proof. We only need to prove (19). We will consider the equations

$$
\begin{gathered}
\delta \mathrm{e}^{\operatorname{ad} H_{0>}}\left(\partial_{\mu n}\right)+\frac{\partial t_{00}}{\partial t_{\mu n}} \log z_{0}+\delta_{\mu 0} z_{0}^{n}=\delta \mathrm{e}^{\operatorname{ad} H}\left(\partial_{\mu n}\right), \\
\delta \mathrm{e}^{\operatorname{ad} H_{i}^{-}}\left(\partial_{\mu n}\right)+\delta_{\mu i}\left(\left(1-\delta_{n 0}\right) z_{i}^{n}+\delta_{n 0} \log z_{i}\right)=\delta \mathrm{e}^{\operatorname{ad} H}\left(\partial_{\mu n}\right), \quad i \in \mathbb{S},
\end{gathered}
$$

which are derived from (5) by taking right logarithmic derivatives.

We take the $p$-derivative of (20) to get

$$
\begin{gathered}
\frac{\mathrm{d}}{\mathrm{d} p}\left[\delta \mathrm{e}^{\operatorname{ad} H_{0>}}\left(\partial_{\mu n}\right)\right]+\left(\frac{\partial t_{00}}{\partial t_{0 n}} \frac{1}{z_{0}}+n \delta_{\mu 0} z_{0}^{n-1}\right) \frac{\mathrm{d} z_{0}}{\mathrm{~d} p}=\frac{\mathrm{d}}{\mathrm{d} p}\left[\delta \mathrm{e}^{\operatorname{ad} H}\left(\partial_{\mu n}\right)\right], \\
\frac{\mathrm{d}}{\mathrm{d} p}\left[\delta \mathrm{e}^{\operatorname{ad} H_{i}^{-}}\left(\partial_{\mu n}\right)\right]+\delta_{\mu i}\left(n\left(1-\delta_{n 0}\right) z_{i}^{n-1}+\delta_{n 0} \frac{1}{z_{i}}\right) \frac{\mathrm{d} z_{i}}{\mathrm{~d} p}=\frac{\mathrm{d}}{\mathrm{d} p}\left[\delta \mathrm{e}^{\operatorname{ad} H}\left(\partial_{\mu n}\right)\right], \quad i \in \mathbb{S} .
\end{gathered}
$$

Now,

$$
\frac{\mathrm{d}}{\mathrm{d} p}\left[\delta \mathrm{e}^{\mathrm{ad} H}\left(\partial_{\mu n}\right)\right]
$$

is analytic in $\overline{\mathbb{C}} \backslash D$ and therefore

$$
0=\oint_{\gamma} \frac{\mathrm{d}}{\mathrm{d} p}\left[\delta \mathrm{e}^{\operatorname{ad} H}\left(\partial_{\mu n}\right)\right] \mathrm{d} p=\sum_{\mu=0}^{M} \oint_{\gamma_{\mu}} \frac{\mathrm{d}}{\mathrm{d} p}\left[\delta \mathrm{e}^{\mathrm{ad} H}\left(\partial_{\mu n}\right)\right] \mathrm{d} p
$$

but from (21) we deduce

$$
\begin{aligned}
& \oint_{\gamma_{0}} \frac{\mathrm{d}}{\mathrm{d} p}\left[\delta \mathrm{e}^{\operatorname{ad} H}\left(\partial_{\mu n}\right)\right] \mathrm{d} p=\oint_{\gamma_{0}} \frac{\mathrm{d}}{\mathrm{d} p}\left[\delta \mathrm{e}^{\operatorname{ad} H_{0>}}\left(\partial_{\mu n}\right)\right] \mathrm{d} p+\oint_{\Gamma_{0}}\left(\frac{\partial t_{00}}{\partial t_{\mu n}} \frac{1}{z_{0}}+n \delta_{\mu 0} z_{0}^{n-1}\right) \mathrm{d} z_{0}, \\
& \oint_{\gamma_{i}} \frac{\mathrm{d}}{\mathrm{d} p}\left[\delta \mathrm{e}^{\operatorname{ad} H}\left(\partial_{\mu n}\right)\right] \mathrm{d} p=\oint_{\gamma_{i}} \frac{\mathrm{d}}{\mathrm{d} p}\left[\delta \mathrm{e}^{\operatorname{ad} H_{i}^{-}}\left(\partial_{\mu n}\right)\right] \mathrm{d} p+\delta_{\mu i} \oint_{\Gamma_{i}}\left(n\left(1-\delta_{n 0}\right) z_{i}^{n-1}+\delta_{n 0} \frac{1}{z_{i}}\right) \mathrm{d} z_{i}, \quad i \in \mathbb{S},
\end{aligned}
$$


where we have changed of variables $z_{\mu}=z_{\mu}(p)$ with $\Gamma_{\mu}=z_{\mu}\left(\gamma_{\mu}\right)$. Now, recalling that

$$
\begin{gathered}
\frac{\mathrm{d}}{\mathrm{d} p}\left[\delta \mathrm{e}^{\mathrm{ad} H_{0>}}\left(\partial_{\mu n}\right)\right]=O\left(p^{-2}\right), \quad p \rightarrow \infty, \\
\frac{\mathrm{d}}{\mathrm{d} p}\left[\delta \mathrm{e}^{\mathrm{ad} H_{i}^{-}}\left(\partial_{\mu n}\right)\right] \text { is holomorphic at } D_{i} \text { for } i \in \mathbb{S}
\end{gathered}
$$

we get

$$
\frac{\partial t_{00}}{\partial t_{\mu n}}=-\left(1-\delta_{\mu 0}\right) \delta_{n 0}
$$

\subsection{Some dispersionless systems within the Whitham hierarchy: the Boyer-Finley-Benney system}

We consider the equations involving the times $\left\{t_{i 0}=: x_{i}, t_{j 1}=: y_{j}, t_{02}=: t\right\}_{i, j=\in \mathbb{S}}$. Now, we write

$$
\Omega_{i 0}=-\log \left(p-q_{i}\right), \Omega_{i 1}=\frac{v_{i}}{p-q_{i}} \text { and } \Omega_{02}=p^{2}-2 w, \text { with } \quad v_{i}:=d_{i-1} \text {, and } w:=-d_{01} .
$$

and the corresponding Whitham equations (13) are

$$
\begin{aligned}
\frac{\partial q_{i}}{\partial y_{j}} & =\frac{\partial v_{j}}{\partial x_{j}}=\frac{\partial}{\partial x}\left(\frac{v_{j}}{q_{i}-q_{j}}\right) \\
\frac{\partial q_{i}}{\partial y_{i}} & =\frac{\partial v_{i}}{\partial x_{i}} \\
\frac{\partial q_{i}}{\partial x_{j}} & =-\frac{\partial \log \left(q_{i}-q_{j}\right)}{\partial x} \\
\frac{\partial q_{i}}{\partial x_{i}} & =-\frac{\partial \log \left(v_{i}\right)}{\partial x} \\
\frac{\partial w}{\partial x_{i}} & =\frac{\partial q_{i}}{\partial x} \\
\frac{\partial q_{i}}{\partial t} & =\frac{\partial\left(q_{i}^{2}-2 w\right)}{\partial x} \\
\frac{\partial v_{i}}{\partial t} & =2 \frac{\partial\left(q_{i} v_{i}\right)}{\partial x} \\
\frac{\partial w}{\partial y_{i}} & =\frac{\partial v_{i}}{\partial x}
\end{aligned}
$$

where $i \neq j$.

Observe that equations (23) and (25) imply

$$
\frac{\partial^{2} \mathrm{e}^{\Phi_{i}}}{\partial x_{i}^{2}}+\frac{\partial^{2} \Phi_{i}}{\partial x \partial y_{i}}=0, \quad \Phi_{i}:=\log v_{i}
$$

which is the Boyer-Finley equation, which appears in General Relativity [4], or dispersionless Toda equation for $\Phi_{i}$, and that equations (27)-(29) form the Benney generalized gas system [32]. 
Notice also that from (24), (22), (26) and (29) we deduce the local existence of a potential function $W$ such that

$$
q_{i}=\frac{\partial W}{\partial x_{i}}, \quad v_{i}=\frac{\partial W}{\partial y_{i}}, \quad w=\frac{\partial W}{\partial x} .
$$

Therefore, this system of equations may be simplified as follows

$$
\begin{aligned}
W_{x_{i} y_{j}}-\left(\frac{W_{y_{j}}}{W_{x_{i}}-W_{x_{j}}}\right)_{x} & =0, & i \neq j, \\
W_{x_{i} x_{j}}+\left(\log \left(W_{x_{i}}-W_{x_{j}}\right)\right)_{x} & =0, & \\
W_{x_{i} x_{i}}+\left(\log \left(W_{y_{i}}\right)\right)_{x} & =0, & \\
W_{x_{i} t}+\left(2 W_{x}-W_{x_{i}}^{2}\right)_{x} & =0, & \\
W_{y_{i} t}-2\left(W_{x_{i}} W_{y_{i}}\right)_{x} & =0, &
\end{aligned}
$$

We stress again that (33) is a form of the Boyer-Finley equation, and that (34) and (35) is a form of Benney system. Therefore, the whole system may be understood as an extension of these equations. This fact, have induce us to propose the name of Boyer-Finley-Benney for the mentioned system.

\subsection{On the existence of a potential for the Whitham hierarchy}

In the previous section we have seen that the Boyer-Finley-Benney equations can be reformulated in terms of a single field. We will show now that this is a general fact for the Whitham hierarchy, being the potential the coefficient

$$
h_{01}=:-(\log \tau)_{x}
$$

as we will see in a forthcoming paper this is essentially due to the existence of a $\tau$-function for the Whitham hierarchy [20].

The Whitham hierarchy is determined in terms of the functions $z_{\mu}$ or its coefficients $d_{\mu n}$ as given in (11). In fact, as was stated in Proposition 1 the coefficients $d_{\mu n}$ are determined in terms of $h_{\mu m}$ and its $x$-derivatives. We will consider inversion formulae for (11)

$$
\begin{aligned}
& p=z_{0}+\sigma_{01} z_{0}^{-1}+\sigma_{02} z_{0}^{-2}+\cdots \\
& p=q_{i}+\sigma_{i 1} z_{i}^{-1}+\sigma_{i 2} z_{i}^{-2}+\cdots
\end{aligned}
$$

where the inversion coefficients $\sigma_{\mu n}$ are polynomials in $d_{\mu m}$, for example

$$
\begin{aligned}
& \sigma_{01}=-d_{01}, \quad \sigma_{02}=-d_{02}, \quad \sigma_{03}=-\left(d_{03}+d_{01}^{2}\right), \\
& \sigma_{i 1}=d_{i-1}, \quad \sigma_{i 2}=d_{i 0} d_{i-1}, \quad \sigma_{i 3}=d_{i-1} d_{i 0}+d_{i-1}^{2} d_{i 1} \text {. }
\end{aligned}
$$

In the following we will use the geometry illustrated in Figure 1. We first show the following

Theorem 2. The following identity holds

$$
\begin{aligned}
& {\left[\delta \mathrm{e}^{\operatorname{ad} H_{0>}}\left(\partial_{\mu n}\right)\right](p)=-\frac{1}{2 \pi \mathrm{i}} \oint_{\Gamma_{\mu}} \log \left(1-\frac{p\left(z_{\mu}\right)}{p}\right) n z_{\mu}^{n-1} \mathrm{~d} z_{\mu}} \\
& \quad+\left(1-\delta_{\mu 0}\right) \delta_{n 0}\left(\log \left(1-\frac{q \mu}{p}\right)-\frac{1}{2 \pi \mathrm{i}} \oint_{\Gamma_{0}} \log \left(1-\frac{p\left(z_{0}\right)}{p}\right) z_{0}^{-1} \mathrm{~d} z_{0}\right), \quad p \in \overline{\mathbb{C}} \backslash D_{0}
\end{aligned}
$$


In the above formula we must understand that when $\mu=0$ the second term of the r.h.s. vanishes even if $q_{0}=\infty$.

Proof. We first introduce

$$
\delta \mathrm{e}^{\mathrm{ad} H_{0>}}\left(\partial_{\mu n}\right)=: \Phi_{\mu n}=\Phi_{\mu n, 1} p^{-1}+\Phi_{\mu n, 2} p^{-2}+\cdots
$$

and observe that

$$
\frac{1}{2 \pi \mathrm{i}} \oint_{\gamma_{0}} p^{m} \frac{\mathrm{d} \Phi_{\mu n}}{\mathrm{~d} p}(p) \mathrm{d} p=-m \Phi_{\mu n, m}, \quad m=1,2, \ldots
$$

Now we consider (20) with the explicit form for $t_{00}$

$$
\begin{aligned}
\delta \mathrm{e}^{\operatorname{ad} H_{0>}}\left(\partial_{\mu n}\right)+\delta_{\mu 0} z_{0}^{n}-\left(1-\delta_{\mu 0}\right) \delta_{n 0} \log z_{0} & =\delta \mathrm{e}^{\operatorname{ad} H}\left(\partial_{\mu n}\right), \\
\delta \mathrm{e}^{\operatorname{ad} H_{i}^{-}}\left(\partial_{\mu n}\right)+\delta_{\mu i}\left(\left(1-\delta_{n 0}\right) z_{i}^{n}+\delta_{n 0} \log z_{i}\right) & =\delta \mathrm{e}^{\operatorname{ad} H}\left(\partial_{\mu n}\right), \quad i \in \mathbb{S}
\end{aligned}
$$

which are derived from (5) by taking right logarithmic derivatives. We act with $p^{m} \frac{\mathrm{d}}{\mathrm{d} p}$ on to get

$$
\begin{array}{r}
p^{m} \frac{\mathrm{d}}{\mathrm{d} p}\left[\delta \mathrm{e}^{\operatorname{ad} H_{0>}}\left(\partial_{\mu n}\right)\right]+p^{m}\left(n \delta_{\mu 0} z_{0}^{n-1}-\left(1-\delta_{\mu 0}\right) \delta_{n 0} z_{0}^{-1}\right) \frac{\mathrm{d} z_{0}}{\mathrm{~d} p}=p^{m} \frac{\mathrm{d}}{\mathrm{d} p}\left[\delta \mathrm{e}^{\operatorname{ad} H}\left(\partial_{\mu n}\right)\right], \\
\left.p^{m} \frac{\mathrm{d}}{\mathrm{d} p}\left[\delta \mathrm{e}^{\operatorname{ad} H_{i}^{-}}\left(\partial_{\mu n}\right)\right]+\delta_{\mu i}\left(n z_{i}^{n-1}+\delta_{n 0} z_{i}^{-1}\right)\right) \frac{\mathrm{d} z_{i}}{\mathrm{~d} p}=p^{m} \frac{\mathrm{d}}{\mathrm{d} p}\left[\delta \mathrm{e}^{\operatorname{ad} H}\left(\partial_{\mu n}\right)\right] .
\end{array}
$$

We observe that

$$
p^{m} \frac{\mathrm{d} \mathfrak{r}}{\mathrm{d} p} \subset \mathfrak{r}
$$

and therefore

$$
0=\oint_{\gamma} p^{m} \frac{\mathrm{d}}{\mathrm{d} p}\left[\delta \mathrm{e}^{\mathrm{ad} H}\left(\partial_{\mu n}\right)\right] p=\sum_{\mu=0}^{M} \oint_{\gamma_{\mu}} p^{m} \frac{\mathrm{d}}{\mathrm{d} p}\left[\delta \mathrm{e}^{\operatorname{ad} H}\left(\partial_{\mu n}\right)\right] .
$$

From (42) we derive

$$
\begin{aligned}
0=\oint_{\gamma_{0}} p^{m} \frac{\mathrm{d}}{\mathrm{d} p} & \left.\delta \mathrm{e}^{\operatorname{ad} H_{0>}}\left(\partial_{\mu n}\right)\right] \mathrm{d} p+\oint_{\Gamma_{0}} p\left(z_{0}\right)^{m}\left(n \delta_{\mu 0} z_{0}^{n-1}-\left(1-\delta_{\mu 0}\right) \delta_{n 0} z_{0}^{-1}\right) \mathrm{d} z_{0} \\
& +\sum_{i=1}^{M}\left(\oint_{\gamma_{i}} p^{m} \frac{\mathrm{d}}{\mathrm{d} p}\left[\delta \mathrm{e}^{\operatorname{ad} H_{i}^{-}}\left(\partial_{\mu n}\right)\right] \mathrm{d} p+\delta_{\mu i} \oint_{\Gamma_{i}} p\left(z_{i}\right)^{m}\left(n z_{i}^{n-1}+\delta_{n 0} z_{i}^{-1}\right) \mathrm{d} z_{i}\right) .
\end{aligned}
$$

Therefore, recalling (40) and

$$
p^{m} \frac{\mathrm{d} \mathfrak{g}_{i}^{-}}{\mathrm{d} p} \subset \mathfrak{g}_{i}^{-}
$$

we may write (43) as follows

$$
m \Phi_{\mu n, m}=\frac{1}{2 \pi \mathrm{i}} \oint_{\Gamma_{\mu}} p\left(z_{\mu}\right)^{m} n z_{\mu}^{n-1} \mathrm{~d} z_{\mu}+\delta_{n 0} \frac{1}{2 \pi \mathrm{i}}\left(\oint_{\Gamma_{\mu}} \frac{p\left(z_{\mu}\right)^{m}}{z_{\mu}} \mathrm{d} z_{\mu}-\oint_{\Gamma_{0}} \frac{p\left(z_{0}\right)^{m}}{z_{0}} \mathrm{~d} z_{0}\right) .
$$

and (36) implies

$$
m \Phi_{\mu n, m}=\frac{1}{2 \pi \mathrm{i}} \oint_{\Gamma_{\mu}} p\left(z_{\mu}\right)^{m} n z_{\mu}^{n-1} \mathrm{~d} z_{\mu}+\left(1-\delta_{\mu 0}\right) \delta_{n 0}\left(q_{\mu}^{m}-\frac{1}{2 \pi \mathrm{i}} \oint_{\Gamma_{0}} \frac{p\left(z_{0}\right)^{m}}{z_{0}} \mathrm{~d} z_{0}\right) .
$$


where it must be understood that when $\mu=0$ the second term of the r.h.s. vanishes. Hence, as

$$
\log \left(1-\frac{q}{p}\right)=-\sum_{m=1}^{\infty} \frac{1}{m} \frac{q^{m}}{p^{m}}, \quad\left|\frac{q}{p}\right|>1,
$$

we immediately derive (39).

As a byproduct of the above proof we get

Corollary 1. The following relation

$$
\sigma_{\mu n}=-\frac{1}{n+\left(1-\delta_{\mu 0}\right) \delta_{n 0}} \frac{\partial(\log \tau)_{x}}{\partial t_{\mu n}}, \quad \sigma_{01}=-(\log \tau)_{x x}
$$

holds.

Proof. We prove the Theorem in the following steps:

1. If we put $m=1 \mathrm{in}(44)$ we get

$$
\frac{\partial h_{01}}{\partial t_{\mu n}}=\frac{1}{2 \pi \mathrm{i}} \oint_{\Gamma_{\mu}}\left(n z_{\mu}^{n-1}+\left(1-\delta_{\mu 0}\right) \delta_{n 0} z_{\mu}^{-1}\right) p\left(z_{\mu}\right) \mathrm{d} z_{\mu}
$$

where we have taken into account that

$$
\oint_{\Gamma_{0}} p\left(z_{0}\right) z_{0}^{-1} \mathrm{~d} z_{0}=0
$$

2. We use the inversion formula (36) in (46) we get

$$
\frac{\partial h_{01}}{\partial t_{\mu n}}=\sum_{l=-1,0,1, \ldots} \frac{1}{2 \pi \mathrm{i}} \oint_{\Gamma_{\mu}}\left(n z_{\mu}^{n-1}+\left(1-\delta_{\mu 0}\right) \delta_{n 0} z_{\mu}^{-1}\right) \sigma_{\mu l} \mathrm{~d} z_{\mu},
$$

and the desired result follows at once.

3. From the identity

$$
\delta \mathrm{e}^{\text {ad } H_{0}^{-}}\left(\frac{\partial}{\partial x}\right)=-\mathrm{e}^{\operatorname{ad} H_{0}^{-}}(p)-p=-z_{0}-p
$$

we get

$$
\frac{\partial h_{01}}{\partial x}=\frac{1}{2 \pi \mathrm{i}} \oint_{\gamma_{0}} p \frac{\mathrm{d} z_{0}}{p} \mathrm{~d} p=\frac{1}{2 \pi \mathrm{i}} \oint_{\Gamma_{0}} p\left(z_{0}\right) \mathrm{d} z_{0}=\sigma_{01}
$$

Observe that all the coefficients $\sigma_{\mu n}$ are determined in terms of $h_{01}$ and its time derivatives. Moreover, as all the coefficients $d_{\mu n}$ are rational functions of the $\sigma_{\mu m}$, for example:

$$
\begin{aligned}
& d_{01}=-\sigma_{01}, \quad d_{02}=-\sigma_{02}, \quad d_{03}=-\sigma_{03}+\sigma_{01}^{2}, \\
& d_{i-1}=\sigma_{i 1}, \quad d_{i 0}=\frac{\sigma_{i 2}}{\sigma_{i 1}}, \quad d_{i 1}=\frac{\sigma_{i 3} \sigma_{i 1}-\sigma_{i 2}^{2}}{\sigma_{i 1}^{3}},
\end{aligned}
$$

all the Lax functions may be written in terms of $h_{01}$ and its $t$-derivatives.

Finally, we may write the contents of Theorem 2 as follows 
Corollary 2. The following identity holds

$$
\begin{aligned}
& {\left[\delta \mathrm{e}^{\operatorname{ad} H_{0>}}\left(\partial_{\mu n}\right)\right](p)=-\frac{1}{2 \pi \mathrm{i}} \oint_{\gamma_{\mu}} \log \left(1-\frac{q}{p}\right) \frac{\mathrm{d} z_{\mu}^{n}}{\mathrm{~d} q} \mathrm{~d} q} \\
& \quad+\left(1-\delta_{\mu 0}\right) \delta_{n 0}\left(\log \left(1-\frac{q_{\mu}}{p}\right)-\frac{1}{2 \pi \mathrm{i}} \oint_{\gamma_{0}} \log \left(1-\frac{q}{p}\right) \frac{\mathrm{d} \log \left(z_{0}(q)\right)}{\mathrm{d} q} \mathrm{~d} q\right), \quad p \in \overline{\mathbb{C}} \backslash D_{0} .
\end{aligned}
$$

For example, if we exclude the times $t_{i 0}$ from the discussion we get the suggesting formula

$$
\left[\delta \mathrm{e}^{\operatorname{ad} H_{0>}}\left(\partial_{\mu n}\right)\right](p)=-\frac{1}{2 \pi \mathrm{i}} \oint_{\gamma_{\mu}} \log \left(1-\frac{q}{p}\right) \frac{\mathrm{d} z_{\mu}^{n}}{\mathrm{~d} q} \mathrm{~d} q
$$

\subsection{Undressing solutions of the Whitham hierarchy}

In $§ 3.1$ we have proved that the differential version of the factorization problem (5) may be described in terms of the Whitham hierarchy. Here we show the equivalence between both descriptions by proving that any solution of the Whitham hierarchy may be formally undressed; i.e., it comes from a convenient factorization problem.

Theorem 3. Any set of Lax functions $z_{\mu}$ and zero-curvature functions $\Omega_{\mu m}$ as in (11)-(12) satisfying the Whitham hierarchy (14), may be obtained by a dressing procedure based in the factorization problem (5) as described in Theorem 1.

Proof. If we take as given the complex numbers $q_{i}^{(0)}$ and the functions $q_{i}, d_{\mu n}$ from Proposition 1 we may determine the coefficients $X_{i}$ and $h_{\mu n}$ up to $x$-independent terms. This last fact is clear from the construction of $z_{\mu}$ as a dressing of $p_{\mu}$. Indeed, we have that $\mathrm{e}^{\text {ad } H_{\mu}^{-}} p_{\mu}:=$ $\mathrm{e}^{\text {ad } \tilde{H}_{\mu}^{-}} \mathrm{e}^{\text {ad } f_{\mu}(p)} p_{\mu}=\mathrm{e}^{\text {ad } \tilde{H}_{\mu}^{-}} p_{\mu}$, where $f_{\mu} \in \mathfrak{c}_{\mu}^{-}$.

We now undress, using the canonical transformation $\mathrm{e}^{- \text {ad } \tilde{H}_{\mu}^{-}}$, the Lax functions and zerocurvature forms: $z_{\mu} \rightarrow p_{\mu}$ and $\Omega_{\mu n} \rightarrow \Omega_{\mu n}^{0}$ with

$$
\Omega_{\mu n}^{0}=\delta \mathrm{e}^{-\operatorname{ad} \tilde{H}_{\mu}^{-}}\left(\partial_{\mu n}\right)+\mathrm{e}^{- \text {ad } \tilde{H}_{\mu}^{-}} \Omega_{\mu n} .
$$

Then,

$$
0=\partial_{\mu n} p_{\nu}=\left\{\Omega_{\mu n}^{0}, p_{\nu}\right\}
$$

and

$$
\frac{\partial \Omega_{\mu n}^{0}}{\partial t_{\nu l}}-\frac{\partial \Omega_{\nu l}^{0}}{\partial t_{\mu n}}+\left\{\Omega_{\mu n}^{0}, \Omega_{\nu l}^{0}\right\}=0
$$

From (49) we deduce that

$$
\Omega_{\mu n, x}^{0}=0
$$

so that (50) implies

$$
\frac{\partial \Omega_{\mu n}^{0}}{\partial t_{\nu l}}=\frac{\partial \Omega_{\nu l}^{0}}{\partial t_{\mu n}}
$$

Moreover, for $n>0$ we have

$$
\Omega_{\mu n}-z_{\mu}^{n} \in \mathfrak{g}_{\mu}^{-} .
$$


Thus, $\mathrm{e}^{- \text {ad } H_{\mu}^{-}} \Omega_{\mu n}-p_{\mu}^{n} \in \mathfrak{g}_{\mu}^{-}$and (48) and (51) allow us to deduce

$$
\begin{gathered}
\Omega_{\mu n}^{0}-p_{\mu}^{n} \in \mathfrak{c}_{\mu}^{-} \subset \mathfrak{g}_{\mu}^{-}, \\
\Omega_{i 0}^{0}+\log \left(p-q_{i}^{(0)}\right) \in \mathfrak{c}_{i}^{-} \subset \mathfrak{g}_{i}^{-} .
\end{gathered}
$$

Hence, recalling (52) we get

$$
\Omega_{\mu n}^{0}=\frac{\partial\left(T_{\mu}+f_{\mu}\right)}{\partial t_{\mu n}}, \quad \text { for some } f_{\mu} \in \mathfrak{c}_{\mu}^{-},
$$

and we can write

$$
\delta \mathrm{e}^{-\operatorname{ad} f_{\mu}}\left(\partial_{\mu m}\right)+\mathrm{e}^{-\operatorname{ad} f_{\mu}} \Omega_{\mu m}^{0}=\delta \mathrm{e}^{\operatorname{ad} T_{\mu}}\left(\partial_{\mu m}\right) .
$$

Therefore, if

$$
H_{\mu}^{-}=C\left(\tilde{H}_{\mu}^{-}, f_{\mu}\right) \in \mathfrak{g}_{\mu}^{-} ; \text {i.e., } \mathrm{e}^{\operatorname{ad} H_{\mu}^{-}}=\mathrm{e}^{\operatorname{ad} \tilde{H}_{\mu}^{-}} \mathrm{e}^{\operatorname{ad} f_{\mu}},
$$

where $C$ was introduced in (6), we have

$$
\Omega_{\mu n}=\delta \mathrm{e}^{\operatorname{ad} H_{\mu}^{-}}\left(\partial_{\mu n}\right)+\mathrm{e}^{\operatorname{ad} H_{\mu}^{-}} \delta \mathrm{e}^{\operatorname{ad} T_{\mu}}\left(\partial_{\mu n}\right)=\delta\left(\mathrm{e}^{\operatorname{ad} H_{\mu}^{-}} \mathrm{e}^{\operatorname{ad} T_{\mu}}\right)\left(\partial_{\mu n}\right), \quad z_{\mu}=\mathrm{e}^{\operatorname{ad} H_{\mu}^{-}} \mathrm{e}^{\operatorname{ad} T_{\mu}} p_{\mu} .
$$

Finally, from definition the zero-curvature connection $\Omega_{\mu n} \in \mathfrak{r}$ and there locally exists $H \in \mathfrak{r}$ such that

$$
\Omega_{\mu n}=\delta \mathrm{e}^{\mathrm{ad} H}\left(\partial_{\mu n}\right),
$$

so that (53) and (54) leads us to the factorization (5) for some $G_{\mu}$.

\subsection{Dressing transformations for the Whitham hierarchy}

In this section we show how to dress any solution of the Whitham hierarchy by using the factorization problem technique. Let $z^{(1)}$ be Lax functions as described in (11), with coefficients denoted by $q_{i}^{(1)}$ and $d_{\mu m}^{(1)}$, and $\Omega_{\mu m}^{(1)}$, as defined in (12), so that the Whitham hierarchy (14) is satisfied:

$$
\frac{\partial z_{\nu}^{(1)}}{\partial t_{\mu n}}=\left\{\Omega_{\mu n}^{(1)}, z_{\nu}^{(1)}\right\} .
$$

Let us assume that $q_{i}^{(1)} \in D_{i}$ so that there exists a Hamiltonian $H^{(1)} \in \mathfrak{r}$ with

$$
\Omega_{\mu n}^{(1)}=\delta \mathrm{e}^{\operatorname{ad} H^{(1)}}\left(\partial_{\mu n}\right)
$$

Given new initial conditions $G_{\mu}, \mu=0,1, \ldots, M$, the factorization problem

$$
\mathrm{e}^{\operatorname{ad} H^{(1)}} \mathrm{e}^{\operatorname{ad} G_{\mu}}=\mathrm{e}^{-\operatorname{ad} H_{\mu}^{-}} \mathrm{e}^{\operatorname{ad} H^{(2)}}, \quad H_{\mu}^{-} \in \mathfrak{g}_{\mu}^{-}, H^{(2)} \in \mathfrak{r},
$$

will lead to a dressing procedure of the solution $z_{\mu}^{(1)}$ of the Whitham hierachy as described below Proposition 2. The new Lax functions

$$
z_{\mu}^{(2)}=\mathrm{e}^{\mathrm{ad} H_{\mu}^{-}} z_{\mu}^{(1)}
$$


are of the form (11) with new coefficients $q_{i}^{(2)}$ and $d_{\mu l}^{(2)}$ determined by $H_{\mu}^{-}$. The functions

$$
\Omega_{\mu m}^{(2)}= \begin{cases}\left(\left(z_{\mu}^{(2)}\right)^{n}\right)_{(\mu,+)}, & n>\delta_{\mu 0}, \\ -\log \left(p-q_{i}^{(2)}\right), & n=0, \mu=i=1, \ldots, M,\end{cases}
$$

(in this case $(\cdot)_{(i,+)}$ projects in the span $\left\{\log \left(p-q_{i}^{(2)}\right),\left(p-q_{i}^{(2)}\right)^{-n}\right\}_{n=1}^{\infty}$ and $(\cdot)_{(0,+)}$ onto the span of $\left.\left\{p^{m}\right\}_{m=0}^{\infty}\right)$ have zero-curvature. Moreover, the Whitham hierarchy

$$
\frac{\partial z_{\nu}^{(2)}}{\partial t_{\mu m}}=\left\{\Omega_{\mu m}^{(2)}, z_{\nu}^{(2)}\right\}
$$

is satisfied.

Proof. We take right logarithmic derivative of (55) to obtain

$$
\delta \mathrm{e}^{\operatorname{ad} H_{\mu}^{-}}\left(\partial_{\nu n}\right)+\mathrm{e}^{\operatorname{ad} H_{\mu}^{-}}\left(\Omega_{\nu n}^{(1)}\right)=\delta \mathrm{e}^{\operatorname{ad} H^{(2)}}\left(\partial_{\nu n}\right)=: \Omega_{\nu n}^{(2)} .
$$

As $\Omega_{\nu n}^{(1)}$ is holomorphic in $D_{\mu}$, for all $\mu \neq \nu$, we deduce that $\Omega_{\nu n}^{(2)}$ is also holomorphic in $D_{\mu}$, $\forall \mu \neq \nu$. When $\mu=\nu$ we have a singular behavior at $p=q_{\nu}^{(1)}$ and we obtain $\Omega_{\nu n}^{(2)}$ with the same structure as in (57). If we write the factor $\mathrm{e}^{\text {ad } H_{\mu}^{-}}$as in Proposition 1 and $X_{i}$ is defined by

$$
\int_{x}^{X_{i}} \frac{\mathrm{d} x}{h_{i 1}(x)}=1
$$

we get, for example, the following coefficients of $z_{\mu}^{(2)}$ :

$$
\begin{aligned}
q_{i}^{(2)} & =\left.X_{i, x} q_{i}^{(1)}\right|_{x=X_{i}}+h_{i 0}, \\
d_{i-1}^{(2)} & =\left.d_{i-1}^{(1)}\right|_{x=X_{i}} X_{i, x} \\
d_{i 0}^{(2)} & =\left.\left(d_{i 0}^{(1)}+h_{i 2, x} d_{i-1}^{(1)}+2 h_{i 2} d_{i-1, x}^{(1)}\right)\right|_{x=X_{i}}, \\
d_{01}^{(2)} & =d_{01}^{(1)}-h_{01, x} .
\end{aligned}
$$

Moreover, the analysis of (56) leads to the proof of all the other properties. For example, from $\Omega_{\mu n}^{(2)}=\delta \mathrm{e}^{\text {ad } H^{(2)}}\left(\partial_{\mu n}\right)$ we deduce the zero curvature condition for the $\left\{\Omega_{\mu n}^{(2)}\right\}$.

Now, we introduce

$$
H^{(0)}:=T(p) \in \mathfrak{r}, \quad T:=\sum_{\mu=0}^{M} T_{\mu}(p)
$$

for which

$$
\Omega_{\mu n}^{(0)}:=\delta \mathrm{e}^{\operatorname{ad} H^{(0)}}\left(\partial_{\mu m}\right)=\left\{\begin{array}{lll}
p^{n}, & \mu=0, \\
-\log \left(p-q_{i}^{(0)}\right), & \mu=i, & n=0 \\
\frac{1}{\left(p-q_{i}^{(0)}\right)^{m}}, & \mu=i, & n \geq 1
\end{array}\right.
$$


for this reason we say that $\mathrm{e}^{\text {ad } H^{(0)}}$ is a vacuum solution of the Whitham hierarchy. Indeed, its dressing

$$
\mathrm{e}^{\operatorname{ad} H^{(0)}} \mathrm{e}^{\operatorname{ad} G_{\mu}}=\mathrm{e}^{-\operatorname{ad} \tilde{H}_{\mu}^{-}} \mathrm{e}^{\text {ad } H^{(1)}}, \quad \tilde{H}_{\mu}^{-} \in \mathfrak{g}_{\mu}^{-}, H^{(1)} \in \mathfrak{r}
$$

- giving $H^{(1)}$ and a new solution $\left\{\Omega_{\mu n}^{(1)}\right\}$ of the Whitham hierarchy - is just the factorization problem (5) when we replace

$$
\mathrm{e}^{\operatorname{ad} \tilde{H}_{\mu}^{-}} \mathrm{e}^{\operatorname{ad}\left(\sum_{\nu \neq \mu} t_{\nu}\right)}=\mathrm{e}^{\operatorname{ad} H_{\mu}^{-}}, \quad H_{\mu}^{-} \in \mathfrak{g}_{\mu}^{-} .
$$

\section{String equations in the Whitham hierarchy}

In this section we study the formulation of the Whitham hierarchy in terms of twistor or string equations and the relation of this formulation with the dressing method described above. We first introduce the Orlov-Schulman operators for the Whitham hierarchy in terms of the factorization problem and then obtain the string equation formulation as a consequence of the factorization problem. In the forthcoming paper [20] we will show that, in fact, the string equations give all solutions of the Whitham hierarchy. Then, string equations and factorization problem are equivalent tools to formulate the Whitham hierarchy. Finally, we introduce a very special class of string equation whose construction is based on centerless Virasoro algebra within the Hamiltonian functions, and therefore we refer to this as the Virasoro class of string equations.

\subsection{Lax and Orlov-Schulman functions of the Whitham hierar- chy}

The Lax functions (10) may be written as

$$
z_{\mu}=\mathrm{e}^{\operatorname{ad} H_{\mu}^{-}} \mathrm{e}^{\operatorname{ad} T_{\mu}} p_{\mu}, \quad \mu=0,1, \ldots, M .
$$

Observe that if we define $\left(p_{\mu}(p), x_{\mu}(x, p)\right)$ by

$$
\left(p_{\mu}, x_{\mu}\right):= \begin{cases}(p, x), & \mu=0 \\ \left(\left(p-q_{i}^{(0)}\right)^{-1},-x\left(p-q_{i}^{(0)}\right)^{2}\right), & \mu=i \in \mathbb{S} .\end{cases}
$$

we have

$$
\left\{p_{\mu}, x_{\mu}\right\}=1 \text {. }
$$

In terms of $x_{\mu}$ the Orlov-Schulman function $m_{\mu}$ is defined as follows

$$
m_{\mu}:=\mathrm{e}^{\operatorname{ad} H_{\mu}^{-}} \mathrm{e}^{\operatorname{ad} T_{\mu}} x_{\mu},
$$

so that is canonically conjugated to $z_{\mu}$; i.e,

$$
\left\{z_{\mu}, m_{\mu}\right\}=1 \text {. }
$$

Notice that the quasi-classical Lax equations also hold for the Orlov-Schulman functions:

$$
\frac{\partial m_{\nu}}{\partial t_{\mu n}}=\left\{\Omega_{\mu n}, m_{\nu}\right\}
$$

We now give a closer look to these functions 
Proposition 3. The Orlov-Schulman functions have the form

$$
m_{\mu}=\sum_{n=1}^{\infty} n t_{\mu n} z_{\mu}^{n-1}+\frac{t_{\mu 0}}{z_{\mu}}+\sum_{n \geq 2} v_{\mu n} z_{\mu}^{-n}, \text { where } t_{01}:=x
$$

and

$$
v_{\mu n+1}= \begin{cases}-X_{i}, & \mu=i=1, \ldots, M, n=0 \\ -\left.\left(n h_{i n}+g_{i n}\left(h_{i n-1}, \ldots, h_{i 2}\right)\right)\right|_{x=X_{i}}, & \mu=i=1, \ldots, M, n>0 \\ -\left(n h_{0 n}+g_{0 n}\left(h_{0 n-1}, \ldots, h_{01}\right)\right), & \mu=0, n \geq 0\end{cases}
$$

being $g_{\mu n}$ differential polynomials.

Proof. From (59) we deduce that

$$
m_{\mu}=\mathrm{e}^{\mathrm{ad} H_{\mu}^{-}}\left(x_{\mu}+\frac{\partial T_{\mu}}{\partial p_{\mu}}\right),
$$

so that

$$
m_{\mu}=\mathrm{e}^{\mathrm{ad} H_{\mu}^{-}} x_{\mu}+\left(1-\delta_{\mu 0}\right) t_{\mu 0} z_{\mu}^{-1}+\sum_{n=1+\delta_{\mu 0}}^{\infty} n t_{\mu n} z_{\mu}^{n-1}
$$

Now, we evaluate

$$
\begin{aligned}
\mathrm{e}^{\mathrm{ad} H_{i}^{-}} x_{i} & =-\left(X_{i}+\left.\sum_{n=2}^{\infty}\left(n h_{i n}+\tilde{g}_{i n}\left(h_{i n-1}, \ldots, h_{i 2}\right)\right)\right|_{x=X_{i}}\left(\frac{p-q_{i}}{X_{i, x}}\right)^{n-1}\right) z_{i}^{-2}, \\
\mathrm{e}^{\text {ad } H_{0}^{-}} x & =x+t_{00} p^{-1}-\sum_{n=1}^{\infty}\left(n h_{0 n}+\tilde{g}_{0 n}\left(h_{0 n-1}, \ldots, h_{01}\right)\right) p^{-n-1}
\end{aligned}
$$

where $\tilde{g}_{\mu n}$ are differential polynomials, but as

$$
\begin{aligned}
\frac{p-q_{i}}{X_{i, x}} & =z_{i}^{-1}+\left.h_{i 1}\right|_{x=X_{i}} z_{i}^{-2}+O\left(z_{i}^{-3}\right), \\
p^{-1} & =z_{0}^{-1}+h_{01}^{\prime} z_{0}^{-3}+O\left(z_{0}^{-4}\right)
\end{aligned}
$$

we get (61).

Observe that the first coefficients of $m_{\mu}$ are

$$
\begin{aligned}
& v_{i 2}=-X_{i}, \\
& v_{i 3}=-\left.2 h_{i, 2}\right|_{x=X_{i}}, \\
& v_{02}=-h_{01} \\
& v_{03}=-2 h_{02} .
\end{aligned}
$$

\subsection{The factorization problem and strings equations}

Let us define new canonical pairs $\left(\hat{z}_{\mu}, \hat{m}_{\mu}\right)$ and $\left(\hat{P}_{\mu}, \hat{Q}_{\mu}\right)$ given by

$$
\begin{aligned}
\hat{z}_{\mu}:=\mathrm{e}^{\operatorname{ad} H_{\mu}^{-}} \mathrm{e}^{\operatorname{ad} T_{\mu}} p, & \hat{P}_{\mu}:=\mathrm{e}^{\operatorname{ad} G_{\mu}} p, \\
\hat{m}_{\mu}:=\mathrm{e}^{\operatorname{ad} H_{\mu}^{-}} \mathrm{e}^{\operatorname{ad} T_{\mu}} x, & \hat{Q}_{\mu}:=\mathrm{e}^{\operatorname{ad} G_{\mu}} x .
\end{aligned}
$$


Observe that

$$
\begin{aligned}
z_{\mu} & =p_{\mu}\left(\hat{z}_{\mu}\right), \\
m_{\mu} & =x_{\mu}\left(\hat{m}_{\mu}, \hat{z}_{\mu}\right),
\end{aligned}
$$

where the functions are defined in (58).

Now, we are ready to give a first version of the string or twistor equations for the Whitham hierarchy:

Proposition 4. For any given solution of the factorization problem (5) with associated canonical pairs $\left(\hat{z}_{\mu}, \hat{m}_{\mu}\right),\left(\hat{P}_{\mu}, \hat{Q}_{\mu}\right)$, as defined in (63), the following string equations hold

$$
\begin{aligned}
& \hat{P}_{\nu}\left(\hat{z}_{\nu}, \hat{m}_{\nu}\right)=\hat{P}_{\mu}\left(\hat{z}_{\mu}, \hat{m}_{\mu}\right) \in \mathfrak{r}, \\
& \hat{Q}_{\nu}\left(\hat{z}_{\nu}, \hat{m}_{\nu}\right)=Q_{\mu}\left(\hat{z}_{\mu}, \hat{m}_{\mu}\right) \in \mathfrak{r} .
\end{aligned}
$$

Proof. The factorization (5) implies

$$
\begin{aligned}
& \hat{P}_{\mu}\left(\hat{z}_{\mu}, \hat{m}_{\mu}\right)=\mathrm{e}^{\text {ad } H_{\mu}^{-}} \mathrm{e}^{\operatorname{ad} T_{\mu}} \mathrm{e}^{\operatorname{ad} G_{\mu}} p=\mathrm{e}^{\operatorname{ad} H} p=\Pi, \\
& \hat{Q}_{\mu}\left(\hat{z}_{\mu}, \hat{m}_{\mu}\right)=\mathrm{e}^{\text {ad } H_{\mu}^{-}} \mathrm{e}^{\operatorname{ad} T_{\mu}} \mathrm{e}^{\operatorname{ad} G_{\mu}} x=\mathrm{e}^{\text {ad } H} x=\Theta .
\end{aligned}
$$

Notice that

$$
\hat{\phi}_{\mu}(p, x):=\left(\hat{P}_{\mu}(p, x), \hat{Q}_{\mu}(p, x)\right)
$$

is a canonical transformation; i.e.,

$$
\left\{\hat{P}_{\mu}, \hat{Q}_{\mu}\right\}=1
$$

that together with (65) ensures that

$$
\hat{\phi}_{\mu}\left(\hat{z}_{\mu}, \hat{m}_{\mu}\right)=\hat{\phi}_{\nu}\left(\hat{z}_{\nu}, \hat{m}_{\nu}\right)=(\Pi, \Theta),
$$

and (64) follows.

The string equations (67) have an interesting interpretation in terms of transition functions between different canonical pairs

$$
\left(\hat{z}_{\mu}, \hat{m}_{\mu}\right)=\hat{\phi}_{\mu \nu}\left(\hat{z}_{\nu}, \hat{m}_{\nu}\right), \quad \hat{\phi}_{\mu \nu}:=\hat{\phi}_{\mu}^{-1} \circ \hat{\phi}_{\nu} .
$$

Now, we define the canonical transformation

$$
\psi_{\mu}(p, x):=\left(p_{\mu}(p), x_{\mu}(p, x)\right)
$$

in terms of which the associated solutions of the Whitham hierarchy are

$$
\left(z_{\mu}, m_{\mu}\right)=\psi_{\mu}\left(\hat{z}_{\mu}, \hat{m}_{\mu}\right)
$$

We also introduce

$$
\phi_{\mu}=\left(P_{\mu}, Q_{\mu}\right):=\hat{\phi}_{\mu} \circ \psi_{\mu}^{-1}, \quad \psi_{\mu}^{-1}=\left(\pi_{\mu}, \theta_{\mu}\right)= \begin{cases}\left(p^{-1}+q_{i}^{(0)},-p^{2} x\right), & \mu=i \in \mathbb{S} \\ (p, x), & \mu=0,\end{cases}
$$

so that

$$
P_{\mu}:=\hat{P}_{\mu}\left(\pi_{\mu}(p, x), \theta_{\mu}(p, x)\right), \quad Q_{\mu}:=\hat{Q}_{\mu}\left(\pi_{\mu}(p, x), \theta_{\mu}(p, x)\right)
$$


and

$$
\left\{P_{\mu}, Q_{\mu}\right\}=1
$$

Observe that this definition is equivalent to

$$
P_{\mu}\left(p_{\mu}, x_{\mu}\right)=\hat{P}_{\mu}(p, x), \quad Q_{\mu}\left(p_{\mu}, x_{\mu}\right)=\hat{Q}_{\mu}(p, x) .
$$

Then, the connection among the different Lax and Orlov-Schulman functions are given by

$$
\left(z_{\mu}, m_{\mu}\right)=\phi_{\mu \nu}\left(z_{\nu}, m_{\nu}\right), \quad \phi_{\mu \nu}:=\psi_{\mu} \circ \hat{\phi}_{\mu \nu} \circ \psi_{\nu}^{-1}=\phi_{\mu}^{-1} \circ \phi_{\nu}
$$

Therefore,

$$
\phi_{\mu}\left(z_{\mu}, m_{\mu}\right)=\phi_{\nu}\left(z_{\nu}, m_{\nu}\right)=(\Pi, \Theta)
$$

and

Proposition 5. Given a solution of (5) and functions $\left(P_{\mu}, Q_{\mu}\right)$ as defined in (69) the string equations

$$
\begin{aligned}
P_{\nu}\left(z_{\nu}, m_{\nu}\right) & =P_{\mu}\left(z_{\mu}, m_{\mu}\right) \in \mathfrak{r}, \\
Q_{\nu}\left(z_{\nu}, m_{\nu}\right) & =Q_{\mu}\left(z_{\mu}, m_{\mu}\right) \in \mathfrak{r}
\end{aligned}
$$

hold $\forall \mu, \nu=0,1, \ldots, M$.

Notice that new initial conditions $\tilde{G}_{\mu}$ of the form

$$
\mathrm{e}^{\operatorname{ad} \tilde{G}_{\mu}}=\mathrm{e}^{\operatorname{ad} G} \mathrm{e}^{\mathrm{ad} G_{\mu}} \text { or } \tilde{G}_{\mu}=C\left(G, G_{\mu}\right),
$$

lead to

$$
\tilde{P}_{\mu}=P\left(P_{\mu}, Q_{\mu}\right), \quad \tilde{Q}_{\mu}=Q\left(P_{\mu}, Q_{\mu}\right) .
$$

Thus, the corresponding string equations are constructed in terms of the initial non-tilded ones.

\subsection{A special class of string equations related to a centreless Virasoro algebra}

Consider the Hamiltonian

$$
G_{\mu}^{(0)}=\frac{x}{\hat{\xi}_{\mu}^{\prime}(p)}
$$

which generate the canonical transformation

$$
(p, x) \rightarrow\left(\hat{f}_{\mu}(p), x / \hat{f}_{\mu}^{\prime}(p)\right), \quad \hat{f}_{\mu}:=\hat{\xi}_{\mu}^{-1}\left(1+\hat{\xi}_{\mu}(p)\right)
$$

Observe that these Hamiltonians close a Lie subalgebra vir $:=\{x f(p), f: \mathbb{C} \rightarrow \mathbb{C}\}$ as $\{x f(p), x g(p)\}=$ $x\left(f^{\prime}(p) g(p)-f(p) g^{\prime}(p)\right) \subset \mathfrak{v i r}$. In fact, $\mathfrak{v i r}$ is a centerless Virasoro algebra with generators

$$
l_{n}:=x p^{n-1}
$$

satisfying

$$
\left\{l_{n}, l_{m}\right\}=(n-m) l_{n+m} .
$$


The functions $\hat{\xi}_{\mu}, \hat{f}_{\mu}$ corresponding to the Virasoro generators (72) are

$$
\hat{\xi}_{\mu}=\left\{\begin{array}{ll}
\frac{p^{2-n}}{2-n}, & n \neq 2, \\
\log p, & n=2,
\end{array} \quad \hat{f}_{\mu}= \begin{cases}(2-n)\left(1+\frac{p^{2-n}}{2-n}\right)^{\frac{1}{2-n}}, & n \neq 2, \\
\mathrm{e} p, & n=2 .\end{cases}\right.
$$

We will also use the harmonic Hamiltonian

$$
R:=\frac{1}{2}\left(p^{2}+x^{2}\right)
$$

which generates the canonical transformation

$$
(p, x) \rightarrow(-x, p)
$$

Let us consider a splitting $\mathbb{S}=I \cup J, I \cap J=\emptyset$, and define the initial conditions

$$
\mathrm{e}^{\operatorname{ad} G_{\mu}}:= \begin{cases}\mathrm{e}^{\operatorname{ad} G_{i}^{(0)}} \mathrm{e}^{\operatorname{ad} R}, & i \in I, \\ \mathrm{e}^{\operatorname{ad} G_{\mu}^{(0)}}, & \mu \in J \cup\{0\},\end{cases}
$$

in terms $G_{\mu}^{(0)}$ as defined in (71). It is easy to realize that

$$
\begin{aligned}
& \left(\hat{P}_{0}, \hat{Q}_{0}\right)=\left(\hat{f}_{0}(p), \frac{x}{\hat{f}_{0}^{\prime}(p)}\right), \\
& \left(\hat{P}_{i}, \hat{Q}_{i}\right)= \begin{cases}\left(-\frac{x}{\hat{f}_{i}^{\prime}(p)}, \hat{f}_{i}(p)\right), & i \in I, \\
\left(\hat{f}_{i}(p), \frac{x}{\hat{f}_{i}^{\prime}(p)}\right), & i \in J,\end{cases}
\end{aligned}
$$

and the corresponding string equations are

$$
\begin{aligned}
& \hat{f}_{0}\left(\hat{z}_{0}\right)=-\frac{\hat{m}_{i}}{\hat{f}_{i}^{\prime}\left(\hat{z}_{i}\right)} \in \mathfrak{r}, \quad \hat{f}_{i}\left(\hat{z}_{i}\right)=\frac{\hat{m}_{0}}{\hat{f}_{0}^{\prime}\left(\hat{z}_{0}\right)} \in \mathfrak{r}, \quad i \in I, \\
& \hat{f}_{0}\left(\hat{z}_{0}\right)=\hat{f}_{i}\left(\hat{z}_{i}\right) \in \mathfrak{r}, \quad \frac{\hat{m}_{i}}{\hat{f}_{i}^{\prime}\left(\hat{z}_{0}\right)}=\frac{\hat{m}_{0}}{\hat{f}_{0}^{\prime}\left(\hat{z}_{0}\right)} \in \mathfrak{r}, \quad i \in J .
\end{aligned}
$$

Taking into account the invariance described in (7) we deduce that he string equations (75) also appear for the following set of initial conditions

$$
\mathrm{e}^{\operatorname{ad} G_{\mu}}:= \begin{cases}\mathrm{e}^{\operatorname{ad} G_{i}^{(0)}}, & i \in I, \\ \mathrm{e}^{\operatorname{ad} G_{\mu}^{(0)}} \mathrm{e}^{-\operatorname{ad} R}, & \mu \in J \cup\{0\} .\end{cases}
$$

where now

$$
\begin{aligned}
& \left(\hat{P}_{0}, \hat{Q}_{0}\right)=\left(\frac{x}{\hat{f}_{0}^{\prime}(p)},-\hat{f}_{0}(p)\right), \\
& \left(\hat{P}_{i}, \hat{Q}_{i}\right)= \begin{cases}\left(\hat{f}_{i}(p), \frac{x}{\hat{f}_{i}^{\prime}(p)}\right), & i \in I, \\
\left(\frac{x}{\hat{f}_{i}^{\prime}(p)},-\hat{f}_{i}(p)\right), & i \in J .\end{cases}
\end{aligned}
$$


We introduce the functions $f_{\mu}$ subject to

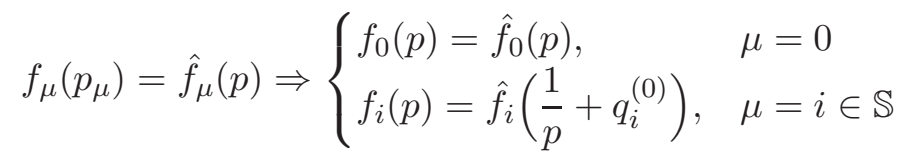

so that

$$
\begin{aligned}
& \left(P_{0}, Q_{0}\right)=\left(-\frac{x}{f_{0}^{\prime}(p)}, f_{0}(p)\right), \\
& \left(P_{i}, Q_{i}\right)= \begin{cases}\left(f_{i}(p),-\frac{x}{f_{i}^{\prime}(p)}\right), & i \in I, \\
\left(-\frac{x}{f_{i}^{\prime}(p)}, f_{i}(p)\right), & i \in J .\end{cases}
\end{aligned}
$$

Therefore, we get the string equations

$$
\begin{aligned}
& f_{0}\left(z_{0}\right)=-\frac{m_{i}}{f_{i}^{\prime}\left(z_{i}\right)} \in \mathfrak{r}, \quad f_{i}\left(z_{i}\right)=\frac{m_{0}}{f_{0}^{\prime}\left(z_{0}\right)} \in \mathfrak{r}, \quad i \in I, \\
& f_{0}\left(z_{0}\right)=f_{i}\left(z_{i}\right) \in \mathfrak{r}, \quad \frac{m_{i}}{f_{i}^{\prime}\left(z_{0}\right)}=\frac{m_{0}}{f_{0}^{\prime}\left(z_{0}\right)} \in \mathfrak{r}, \quad i \in J .
\end{aligned}
$$

These string equations reduces to Krichever type of string equations considered in [12] for $J=\mathbb{S}$ and to the Takasaki-Takebe type [27] for $J=\emptyset$.

\section{Additional symmetries for the Whitham hierarchy}

This section is devoted to the analysis of the additional or master symmetries of the Whitham hierarchy. For that aim we we characterize the additional symmetries in terms of deformations of the factorization problem (5). We compute then some explicit examples of additional symmetries leading to functional symmetries of the generalized Benney gas equations. Finally, we study its action on Virasoro string equations.

\subsection{Deformation of the factorization problem and additional symmetries}

The treatment of functional symmetries of dispersionless hierarchies as additional symmetries was first given in [17] for the dispersionless KP hierarchy. Then, its formulation as a deformation of a factorization problem for the $r$-th dispersionless Toda hierarchy was considered in [16].

In this section we allow each initial condition Hamiltonian $G_{\mu}$ to depend on a external parameter $s$

$$
G_{\mu}:=G_{\mu}(s) .
$$

Then, the factorization problem (5) also depends on $s$

$$
\mathrm{e}^{\operatorname{ad} T_{\mu}} \mathrm{e}^{\operatorname{ad} G_{\mu}(s)}=\mathrm{e}^{-\operatorname{ad} H_{\mu}^{-}(s)} \mathrm{e}^{\operatorname{ad} H(s)}, \text { with } H_{\mu}^{-}(s) \in \mathfrak{g}_{\mu}^{-} \text {and } H(s) \in \mathfrak{r} .
$$

Thus, we deduce that 
Theorem 4. Additional symmetries of the Whitham hierarchy are characterized by functions $F_{\mu}\left(z_{\mu}, m_{\mu}\right)$ as follows

$$
\begin{gathered}
\frac{\partial z_{\nu}}{\partial s}=-\frac{\partial F_{\nu}}{\partial m_{\nu}}+\sum_{\mu=0}^{M}\left\{\left(F_{\mu}\left(z_{\mu}, m_{\mu}\right)\right)_{(\mu,+)}, z_{\nu}\right\}, \\
\frac{\partial m_{\nu}}{\partial s}=-\frac{\partial F_{\nu}}{\partial z_{\nu}}+\sum_{\mu=0}^{M}\left\{\left(F_{\mu}\left(z_{\mu}, m_{\mu}\right)\right)_{(\mu,+)}, m_{\nu}\right\} .
\end{gathered}
$$

Proof. Taking the right logarithmic derivative of (79) with respect to $s$ we get

$$
\delta \mathrm{e}^{\operatorname{ad} H_{\mu}^{-}}\left(\frac{\partial}{\partial s}\right)+F_{\mu}\left(z_{\mu}, m_{\mu}\right)=\delta \mathrm{e}^{\operatorname{ad} H}\left(\frac{\partial}{\partial s}\right),
$$

where

$$
F_{\mu}\left(z_{\mu}, m_{\mu}\right)=\hat{F}_{\mu}\left(\hat{z}_{\mu}, \hat{m}_{\mu}\right), \quad \hat{F}_{\mu}:=\delta \mathrm{e}^{\operatorname{ad} G_{\mu}}\left(\frac{\partial}{\partial s}\right) .
$$

Observe that from the splitting

$$
F_{\mu}=F_{\mu}^{-}+F, \quad F:=\sum_{\nu=0}^{M} F_{(\nu,+)}
$$

with

$$
F_{\mu}^{-} \in \mathfrak{g}_{\mu}^{-}, \quad F \in \mathfrak{r},
$$

and from (80) we get that

$$
\begin{aligned}
\delta \mathrm{e}^{\operatorname{ad} H_{\mu}^{-}}\left(\frac{\partial}{\partial s}\right) & =-F_{\mu}^{-}=F-F_{\mu}, \\
\delta \mathrm{e}^{\operatorname{ad} H}\left(\frac{\partial}{\partial s}\right) & =F .
\end{aligned}
$$

Therefore, from

$$
\frac{\partial z_{\mu}}{\partial s}=\left\{\delta \mathrm{e}^{\operatorname{ad} H_{\mu}^{-}}\left(\frac{\partial}{\partial s}\right), z_{\mu}\right\}, \quad \frac{\partial m_{\mu}}{\partial s}=\left\{\delta \mathrm{e}^{\operatorname{ad} H_{\mu}^{-}}\left(\frac{\partial}{\partial s}\right), m_{\mu}\right\}
$$

we get the desired result.

An important reduction is given by $t_{\mu n}=0$ for $n>N_{\mu}$. If we assume that

$$
F_{\mu}\left(z_{\mu}, m_{\mu}\right)=c_{\mu} \log z_{\mu}+\sum_{i, j \in \mathbb{Z}} c_{\mu, i j} z_{\mu}^{i} m_{\mu}^{j}
$$

and

$$
m_{\mu}=\sum_{n=1}^{N_{\mu}} n t_{\mu n} z_{\mu}^{n-1}+\frac{t_{\mu 0}}{z_{\mu}}+\sum_{n \geq 2} v_{\mu n} z_{\mu}^{-n},
$$

imposing $F_{\mu}\left(z_{\mu}, m_{\mu}\right)$ to have no terms proportional to $z_{\mu}^{n}$ for $n>N_{\mu}$, we ensure that the constraints are preserve. We request this for each of the products $z_{\mu}^{i} m_{\mu}^{j}$ :

$$
\begin{aligned}
z_{\mu}^{i} m_{\mu}^{j} & =z_{\mu}^{i}\left(N_{\mu} t_{\mu N_{\mu}} z_{\mu}^{N_{\mu}-1}+\cdots+t_{\mu 1}+t_{\mu 0} z_{\mu}^{-1}+v_{\mu 2} z_{\mu}^{-2}+v_{\mu 3} z_{\mu}^{-3}+\ldots\right)^{j} \\
& =\left(N_{\mu} t_{\mu N_{\mu}}\right)^{j} z_{\mu}^{i+\left(N_{\mu}-1\right) j}+\cdots \Rightarrow c_{\mu, i j}=0 \text { if } i+\left(N_{\mu}-1\right) j>N_{\mu} .
\end{aligned}
$$


Hence,

$$
F_{\mu}\left(z_{\mu}, m_{\mu}\right)=c_{\mu} \log z_{\mu}+\sum_{n=1}^{N_{\mu}} \alpha_{\mu n}\left(\frac{m_{\mu}}{N_{\mu} z_{\mu}^{N_{\mu}-1}}\right) z_{\mu}^{n}
$$

with $\alpha_{n}$ being analytic functions.

Some times is convenient to consider that only one of the initial conditions is deformed, say the $\alpha$-component:

$$
\frac{\partial G_{\mu}}{\partial s}=\delta_{\mu \alpha} \frac{\partial G_{\alpha}}{\partial s}, \quad \forall \mu=0,1, \ldots, M .
$$

In this case we get the following symmetry equations

$$
\begin{aligned}
\frac{\partial z_{\nu}}{\partial s} & =\delta_{\nu \alpha} \frac{\partial F_{\alpha}}{\partial m_{\alpha}}+\left\{\left(F_{\alpha}\left(z_{\alpha}, m_{\alpha}\right)\right)_{(\mu,+)}, z_{\nu}\right\}, \\
\frac{\partial m_{\nu}}{\partial s} & =-\delta_{\nu \alpha} \frac{\partial F_{\alpha}}{\partial z_{\alpha}}+\left\{\left(F_{\alpha}\left(z_{\alpha}, m_{\alpha}\right)\right)_{(\mu,+)}, m_{\nu}\right\} .
\end{aligned}
$$

\subsection{Action of additional symmetries on the potential function}

Observe that if we express $m_{\mu}=m_{\mu}\left(\boldsymbol{t}, z_{\mu}\right)$ we get $F_{\mu}\left(z_{\mu}, m_{\mu}\left(z_{\mu}\right)\right)=: f_{\mu}\left(z_{\mu}\right)$. Then, inspired by Theorem 1, we get

Theorem 5. The following relation

$$
\frac{\partial(\log \tau)_{x}}{\partial s}=-\frac{1}{2 \pi \mathrm{i}} \sum_{\mu=0}^{M} \oint_{\Gamma_{\mu}} p\left(z_{\mu}\right) \frac{\mathrm{d} f_{\mu}}{\mathrm{d} z_{\mu}} \mathrm{d} z_{\mu}
$$

holds.

Proof. From (80) and

$$
0=\oint_{\gamma} p \frac{\mathrm{d}}{\mathrm{d} p}\left[\delta \mathrm{e}^{\mathrm{ad} H}\left(\frac{\partial}{\partial s}\right)\right] \mathrm{d} p=\sum_{\nu=0}^{M} \oint_{\gamma_{\nu}} p \frac{\mathrm{d}}{\mathrm{d} p}\left[\delta \mathrm{e}^{\mathrm{ad} H}\left(\frac{\partial}{\partial s}\right)\right] \mathrm{d} p
$$

we conclude that

$$
0=\sum_{\nu=0}^{M}\left[\oint_{\gamma_{\nu}} p \frac{\mathrm{d}}{\mathrm{d} p}\left[\delta \mathrm{e}^{\mathrm{ad} H_{\nu}^{-}}\left(\frac{\partial}{\partial s}\right)\right] \mathrm{d} p+\oint_{\gamma_{\nu}} p \frac{\mathrm{d}}{\mathrm{d} p}\left[f_{\nu}\left(z_{\nu}\right)\right] \mathrm{d} p\right] .
$$

But

$$
p \frac{\mathrm{d}}{\mathrm{d} p}\left[\delta \mathrm{e}^{\mathrm{ad} H_{i}^{-}}\left(\frac{\partial}{\partial s}\right)\right] \in \mathfrak{g}_{i}^{-}
$$

is holomorphic in $D_{i}$ and

$$
p \frac{\mathrm{d}}{\mathrm{d} p}\left[\delta \mathrm{e}^{\text {ad } H_{0}^{-}}\left(\frac{\partial}{\partial s}\right)\right]=-\frac{\partial h_{01}}{\partial s} p^{-1}+O\left(p^{-2}\right)+\frac{\partial t_{00}}{\partial s} z_{0}^{-1} p \frac{\mathrm{d} z_{0}}{\mathrm{~d} p},
$$

and the stated result follows. 
Let us assume the expansion

$$
f_{\mu}=\sum_{n=-\infty}^{\infty} f_{\mu n} z_{\mu}^{n}
$$

and perform a change of variables $p \rightarrow z_{\mu}$ to get

$$
\begin{aligned}
\frac{\partial h_{01}}{\partial s} & =\frac{1}{2 \pi \mathrm{i}} \sum_{\mu=0}^{M} \oint_{\Gamma_{\mu}}\left(\sum_{n=-\infty}^{\infty} f_{\mu n} n z_{\mu}^{n-1}\right)\left(\sum_{\substack{l=-1,0,1, \ldots \\
\mu=0, \ldots, M \\
n=-1,0,1, \ldots}} n f_{\mu n} \sigma_{\mu n}=-f_{0-1}+\sum_{\substack{\mu=0, \ldots, M \\
n \geq 1}} n f_{\mu n} \sigma_{\mu n}\right. \\
& =\sum_{\substack{n \\
n}} z_{\mu}
\end{aligned}
$$

Therefore, (47) gives

$$
\frac{\partial h_{01}}{\partial s}=-f_{0-1}+f_{01} \frac{\partial h_{01}}{\partial x}+\sum_{\substack{\mu=0, \ldots, M \\ n \geq 1+\delta_{\mu 0}}} f_{\mu n} \frac{\partial h_{01}}{\partial t_{\mu n}}
$$

This will be a linear PDE for $h_{01}$ if we ensure that the coefficients $f_{\mu n}$ that the dependence on the functions $v_{\mu n}$ is restricted to $v_{02}=-h_{01}$, i.e. do not depend on rational functions of $h_{01}$ and its derivatives. This is the case always for the time reduction $t_{\mu n}=0$ for $n>N_{\mu}$, $\forall \mu=0,1, \ldots, M$. For example, we take

$$
F_{\mu}=\alpha_{\mu}\left(\frac{m_{\mu}}{N_{\mu} z_{\mu}^{N_{\mu}-1}}\right) z_{\mu}^{n}, \quad n=1, \ldots, N_{\mu}
$$

Then,

$$
\frac{m_{\mu}}{N_{\mu} z_{\mu}^{N_{\mu}-1}}=t_{\mu N_{\mu}}+\frac{N_{\mu}-1}{N_{\mu}} t_{\mu N_{\mu}-1} z_{\mu}^{-1}+\cdots+\frac{1}{N_{\mu}} t_{\mu 0} z_{\mu}^{-N_{\mu}}+\frac{1}{N_{\mu}} v_{\mu 2} z_{\mu}^{-N_{\mu}-1}+\cdots,
$$

and therefore

$$
\alpha_{\mu}\left(\frac{m_{\mu}}{N_{\mu} z_{\mu}^{N_{\mu}-1}}\right)=A_{\mu 0}+A_{\mu 1} z_{\mu}^{-1}+\cdots
$$

with

$$
\begin{aligned}
A_{\mu 0} & =\alpha_{\mu}\left(t_{\mu N_{\mu}}\right) \\
A_{\mu 1} & =\alpha_{\mu}^{\prime}\left(t_{\mu N_{\mu}}\right) \frac{N_{\mu}-1}{N_{\mu}} t_{\mu N_{\mu}-1}, \\
A_{\mu 2} & =\alpha_{\mu}^{\prime}\left(t_{\mu N_{\mu}}\right) \frac{N_{\mu}-2}{N_{\mu}} t_{\mu N_{\mu}-2}+\frac{1}{2} \alpha_{\mu}^{\prime \prime}\left(t_{\mu N_{\mu}}\right) \frac{\left(N_{\mu}-1\right)^{2}}{N_{\mu}^{2}} t_{\mu N_{\mu}-1}^{2}, \\
& \vdots \\
A_{\mu N_{\mu}} & =\alpha_{\mu}^{\prime}\left(t_{\mu N_{\mu}}\right) \frac{t_{\mu 0}}{N_{\mu}}+\alpha_{\mu}^{\prime \prime}\left(t_{\mu N_{\mu}}\right) \frac{\sum_{r+s=N_{\mu}}^{\prime} t_{\mu r} t_{\mu s}}{N_{\mu}}+\cdots+\alpha_{\mu}^{\left(N_{\mu}\right)}\left(t_{\mu} N_{\mu}\right) \frac{\left(N_{\mu}-1\right)^{N_{\mu}}}{N_{\mu} ! N_{\mu}^{N_{\mu}}} t_{\mu N_{\mu}-1}^{N_{\mu}}, \\
A_{\mu N_{\mu}+1} & =\alpha_{\mu}^{\prime}\left(t_{\mu N_{\mu}}\right) \frac{v_{\mu 2}}{N_{\mu}}+\alpha_{\mu}^{\prime \prime}\left(t_{\mu N_{\mu}}\right) \frac{\sum_{r+s=N_{\mu}+1}^{\prime} t_{\mu r} t_{\mu s}}{N_{\mu}}+\cdots+\alpha_{\mu}^{\left(N_{\mu}+1\right)}\left(t_{\mu} N_{\mu}\right) \frac{\left(N_{\mu}-1\right)^{N_{\mu}+1}}{\left(N_{\mu}+1\right) ! N_{\mu}^{N_{\mu}+1}} t_{\mu N_{\mu}-1}^{N_{\mu}+1},
\end{aligned}
$$


Here $\sum^{\prime}$ means that if $r=s$ then we multiply this contribution by $1 / 2$. We see that all the coefficients $A_{\mu 0}, \ldots, A_{\mu} N_{\mu}$ do not depend on the functions $v_{\mu 2}$, for all the others the coefficients $v_{\mu n}$ contribute. In particular, in $A_{0 N_{0}+1}$ depends on $v_{02}$.

We have the formula

$$
f_{\mu m}=A_{\mu n-m}
$$

and (86) reads

$$
\frac{\partial h_{01}}{\partial s}=-f_{0-1}+f_{01} \frac{\partial h_{01}}{\partial x}+\sum_{\substack{\mu=0, \ldots, M \\ 1+\delta_{\mu 0} \leq m \leq n}} A_{\mu n-m} \frac{\partial h_{01}}{\partial t_{\mu n}}
$$

For $n=1, \ldots, N_{\mu}-1$ the coefficients $A$ 's that appear in the above equations do not depend on any $v$ 's, $v_{02}=-h_{01}$ and for $\mu=0, n=N_{0}$ the coefficient $A_{0 N_{0}+1}$ do depend linearly on $v_{02}=-h_{01}$.

Notice that (88) and (87) allows us to describe the motion of the potential $h_{01}$ of the Whitham hierarchy under additional symmetries via a linear PDEs.

\subsection{Functional symmetries of the Boyer-Finley-Benney system}

Let us take $N_{0}=2$ and $N_{i}=1$, so that the involved times are $\left\{t_{i 0}=: x_{i}, t_{j 1}=: y_{j}, t_{02}=: t\right\}_{i, j=1}^{M}$ and the PDE' system is the one presented in $\S 3.2$. Now, we have

$$
\begin{aligned}
m_{0} & =2 t z_{0}+x+t_{00} z_{0}^{-1}+v_{02} z_{0}^{-2}+\cdots, \quad t_{00}=x_{1}+\cdots+x_{M} \\
m_{i} & =y_{i}+x_{i} z_{i}^{-1}+v_{i 2} z_{i}^{-2}+\cdots,
\end{aligned}
$$

so that

$$
\begin{aligned}
\alpha_{0}\left(\frac{m_{0}}{2 z_{0}}\right) & =\alpha_{0}(t)+\alpha_{0}^{\prime}(t) \frac{x}{2} z_{0}^{-1}+\left(\alpha_{0}^{\prime}(t) \frac{t_{00}}{2}+\alpha_{0}^{\prime \prime}(t) \frac{x^{2}}{8}\right) z_{0}^{-2}+\cdots, \\
\alpha_{i}\left(m_{i}\right) & =\alpha_{i}\left(y_{i}\right)+\alpha_{i}^{\prime}\left(y_{i}\right) x_{i} z_{i}^{-1}+\left(\alpha_{i}^{\prime}\left(y_{i}\right) v_{i 2}+\alpha_{i}^{\prime \prime}\left(y_{i}\right) \frac{x_{i}^{2}}{2}\right) z_{i}^{-2}+\cdots,
\end{aligned}
$$

We put $C_{\mu}=0$ as these symmetries corresponds to the first flows $\frac{\partial}{\partial x_{i}}$. Then, in the context of (85) we have three different type of generators:

$$
\begin{aligned}
F_{0}^{(1)} & =\alpha_{0}\left(\frac{m_{0}}{2 z_{0}}\right) z_{0}=\alpha_{0}(t) z_{0}+\alpha_{0}^{\prime}(t) \frac{x}{2}+\left(\alpha_{0}^{\prime}(t) \frac{t_{00}}{2}+\alpha_{0}^{\prime \prime}(t) \frac{x^{2}}{8}\right) z_{0}^{-1}+\cdots, \\
F_{0}^{(2)} & =\alpha_{0}\left(\frac{m_{0}}{2 z_{0}}\right) z_{0}^{2}=\alpha_{0}(t) z_{0}^{2}+\alpha_{0}^{\prime}(t) \frac{x}{2} z_{0}+\left(\alpha_{0}^{\prime}(t) \frac{t_{00}}{2}+\alpha_{0}^{\prime \prime}(t) \frac{x^{2}}{8}\right)+\cdots, \\
F_{i} & =\alpha_{i}\left(m_{i}\right) z_{i}=\alpha_{i}\left(y_{i}\right) z_{i}+\alpha_{i}^{\prime}\left(y_{i}\right) x_{i} z_{i}^{-1}+\left(\alpha_{i}^{\prime}\left(y_{i}\right) v_{i 2}+\alpha_{i}^{\prime \prime}\left(y_{i}\right) \frac{x_{i}^{2}}{2}\right) z_{i}^{-2}+\cdots .
\end{aligned}
$$

Therefore,

$$
\begin{aligned}
\frac{\partial F_{0}^{(1)}}{\partial m_{0}} & =\frac{1}{2} \alpha_{0}^{\prime}\left(\frac{m_{0}}{2 z_{0}}\right), \\
\frac{\partial F_{0}^{(2)}}{\partial m_{0}} & =\frac{1}{2} \alpha_{0}^{\prime}\left(\frac{m_{0}}{2 z_{0}}\right) z_{0} \\
\frac{\partial F_{i}}{\partial m_{i}} & =\alpha_{i}^{\prime}\left(m_{i}\right) z_{i}
\end{aligned}
$$


and

$$
\begin{aligned}
\left(F_{0}^{(1)}\right)_{(0,+)} & =\alpha_{0}(t) \Omega_{01}+\alpha_{0}^{\prime}(t) \frac{x}{2}, \\
\left(F_{0}^{(2)}\right)_{(0,+)} & =\alpha_{0}(t) \Omega_{02}+\alpha_{0}^{\prime}(t) \frac{x}{2} \Omega_{01}+\alpha_{0}^{\prime}(t) \frac{t_{00}}{2}+\alpha_{0}^{\prime \prime}(t) \frac{x^{2}}{8}, \\
\left(F_{i}\right)_{(i,+)} & =\alpha_{i}\left(y_{i}\right) \Omega_{i 1} .
\end{aligned}
$$

Hence, the evolution of the Lax functions under these three types of symmetries is characterized by the following PDE' system

$$
\begin{aligned}
& S_{0}^{(1)}:\left\{\begin{array}{l}
\frac{\partial z_{0}}{\partial s_{0}^{(1)}}=\frac{1}{2} \alpha_{0}^{\prime}\left(\frac{m_{0}}{2 z_{0}}\right)+\alpha_{0}(t) \frac{\partial z_{0}}{\partial x}-\frac{1}{2} \alpha_{0}^{\prime}(t) \frac{\partial z_{0}}{\partial p}, \\
\frac{\partial z_{i}}{\partial s_{0}^{(1)}}=\alpha_{0}^{\prime}(t) \frac{\partial z_{i}}{\partial x}-\frac{1}{2} \alpha_{0}^{\prime}(t) \frac{\partial z_{i}}{\partial p},
\end{array}\right. \\
& S_{0}^{(2)}:\left\{\begin{array}{l}
\frac{\partial z_{0}}{\partial s_{0}^{(2)}}=\frac{1}{2} \alpha_{0}^{\prime}\left(\frac{m_{0}}{2 z_{0}}\right) z_{0}+\alpha_{0}(t) \frac{\partial z_{0}}{\partial t}+\frac{1}{2} \alpha_{0}^{\prime}(t) x \frac{\partial z_{0}}{\partial x}-\frac{1}{2} \alpha_{0}^{\prime}(t) p \frac{\partial z_{0}}{\partial p}-\alpha_{0}^{\prime \prime}(t) \frac{x}{4} \frac{\partial z_{0}}{\partial p}, \\
\frac{\partial z_{i}}{\partial s_{0}^{(2)}}=\alpha_{0}(t) \frac{\partial z_{i}}{\partial t}+\frac{1}{2} \alpha_{0}^{\prime}(t) x \frac{\partial z_{i}}{\partial x}-\frac{1}{2} \alpha_{0}^{\prime}(t) p \frac{\partial z_{i}}{\partial p}-\alpha_{0}^{\prime \prime}(t) \frac{x}{4} \frac{\partial z_{i}}{\partial p},
\end{array}\right. \\
& S_{i}:\left\{\begin{array}{l}
\frac{\partial z_{0}}{\partial s_{i}}=\alpha_{i}\left(y_{i}\right) \frac{\partial z_{0}}{\partial y_{i}}, \\
\frac{\partial z_{j}}{\partial s_{i}}=\alpha_{i}\left(y_{i}\right) \frac{\partial z_{j}}{\partial y_{i}}, \\
\frac{\partial z_{i}}{\partial s_{i}}=\alpha_{i}^{\prime}\left(m_{i}\right) z_{i}+\alpha_{i}\left(y_{i}\right) \frac{\partial z_{i}}{\partial y_{i}},
\end{array}\right.
\end{aligned}
$$

We now analyze how the dependent variables $\left\{w, v_{i}, q_{i}\right\}_{i=1}^{M}$ evolve under these symmetries

- The $S_{0}^{(1)}$ equations (89) implies a transformation that only involves the independent variables $(x, t)$ characterized by the following PDEs

$$
\begin{aligned}
\frac{\partial w}{\partial s_{0}^{(1)}}-\alpha_{0}(t) \frac{\partial w}{\partial x}+\frac{x}{4} \alpha_{0}^{\prime \prime}(t) & =0 \\
\frac{\partial v_{i}}{\partial s_{0}^{(1)}}-\alpha_{0}(t) \frac{\partial v_{i}}{\partial x} & =0 \\
\frac{\partial q_{i}}{\partial s_{0}^{(1)}}-\alpha_{0}(t) \frac{\partial q_{i}}{\partial x}+\frac{1}{2} \alpha_{0}^{\prime}(t) & =0
\end{aligned}
$$

and the symmetry transformation is

$$
\begin{aligned}
& w(x, t) \rightarrow w(x+f(t), t)-\frac{f^{\prime \prime}(t)}{4}\left(x+\frac{f(t)}{2}\right), \\
& v_{i}(x, t) \rightarrow v_{i}(x+f(t), t), \\
& q_{i}(x, t) \rightarrow q_{i}(x+f(t), t)-\frac{f^{\prime}(t)}{2}
\end{aligned}
$$

with $f:=s_{0}^{(1)} \alpha_{0}$. For the potential $W$ this symmetry reads

$$
W(x, t) \rightarrow W(x+f(t), t)-\frac{f^{\prime \prime}(t)}{8} x(x+f(t))-\frac{f^{\prime}(t)}{2} \sum_{i=1}^{M} x_{i} .
$$


- In this case the $S_{0}^{(2)}$ equations (90) implies a transformation characterized by the following PDEs

$$
\begin{array}{r}
\frac{\partial w}{\partial s_{0}^{(2)}}-\alpha_{0}(t) \frac{\partial w}{\partial t}-\frac{1}{2} \alpha_{0}^{\prime}(t) x \frac{\partial w}{\partial x}-\alpha_{0}^{\prime}(t) w+\frac{t_{00}}{4} \alpha_{0}^{\prime \prime}(t)+\frac{x^{2}}{16} \alpha_{0}^{\prime \prime \prime}(t)=0 \\
\frac{\partial v_{i}}{\partial s_{0}^{(2)}}-\alpha_{0}(t) \frac{\partial v_{i}}{\partial t}-\frac{1}{2} \alpha_{0}^{\prime}(t) x \frac{\partial v_{i}}{\partial x}-\frac{1}{2} \alpha_{0}^{\prime}(t) v_{i}=0 \\
\frac{\partial q_{i}}{\partial s_{0}^{(2)}}-\alpha_{0}(t) \frac{\partial q_{i}}{\partial t}-\frac{1}{2} \alpha_{0}^{\prime}(t) x \frac{\partial q_{i}}{\partial x}-\frac{1}{2} \alpha_{0}^{\prime}(t) q_{i}-\frac{1}{4} \alpha_{0}^{\prime \prime}(t) x=0,
\end{array}
$$

and the symmetry transformation is

$$
\begin{aligned}
& w(x, t) \rightarrow T^{\prime}(t) w\left(\sqrt{T^{\prime}(t)} x, T(t)\right)-\frac{t_{00}}{4} \frac{T^{\prime \prime}(t)}{T^{\prime}(t)}+\frac{1}{16}\{T, t\}_{\mathrm{S}} x^{2}, \\
& v_{i}(x, t) \rightarrow \sqrt{T^{\prime}(t)} v_{i}\left(\sqrt{T^{\prime}(t)} x, T(t)\right), \\
& q_{i}(x, t) \rightarrow \sqrt{T^{\prime}(t)} q_{i}\left(\sqrt{T^{\prime}(t)} x, T(t)\right)+\frac{1}{4} \frac{T^{\prime \prime}(t)}{T^{\prime}(t)} x
\end{aligned}
$$

with $T:=T\left(s_{0}^{(2)}, t\right)$ characterized by the following relation

$$
\int_{t}^{T} \frac{\mathrm{d} t}{\alpha_{0}(t)}=s_{0}^{(2)}
$$

and we have used the Schwarztian derivative

$$
\{T, t\}_{\mathrm{S}}:=\left(\frac{T^{\prime \prime}(t)}{T^{\prime}(t)}\right)^{\prime}-\frac{1}{2}\left(\frac{T^{\prime \prime}(t)}{T^{\prime}(t)}\right)^{2}=\frac{T^{\prime \prime \prime}(t)}{T^{\prime}(t)}-\frac{3}{2}\left(\frac{T^{\prime \prime}(t)}{T^{\prime}(t)}\right)^{2},
$$

which must be not confused with the Poisson bracket.

For the potential $W$ this symmetry reads

$$
W(x, t) \rightarrow \sqrt{T^{\prime}(t)} W\left(\sqrt{T^{\prime}(t)} x, T(t)\right)+\frac{1}{4} \frac{T^{\prime \prime}(t)}{T^{\prime}(t)} x \sum_{i=1}^{M} x_{i}+\frac{1}{48}\{T, t\}_{\mathrm{S}} x^{3} .
$$

- The $S_{i}$-symmetry characterized by equations (91) implies a transformation that only involves the independent variables $\left(x, y_{i}\right)$ as follows

$$
\begin{array}{rlr}
\frac{\partial w}{\partial s_{i}}-\alpha_{i}\left(y_{i}\right) \frac{\partial w}{\partial y_{i}} & =0, & \\
\frac{\partial v_{j}}{\partial s_{i}}-\alpha_{i}\left(y_{i}\right) \frac{\partial v_{j}}{\partial y_{i}} & =0, & j \neq i \\
\frac{\partial v_{i}}{\partial s_{i}}-\alpha_{i}\left(y_{i}\right) \frac{\partial v_{i}}{\partial y_{i}}-\alpha_{i}^{\prime}\left(y_{i}\right) v_{i} & =0, \\
\frac{\partial q_{j}}{\partial s_{i}}-\alpha_{i}\left(y_{i}\right) \frac{\partial q_{j}}{\partial y_{i}} & =0,
\end{array}
$$

Thus, if $Y_{i}\left(s_{i}, y_{i}\right)$ is defined by

$$
\int_{y_{i}}^{Y_{i}} \frac{\mathrm{d} y_{i}}{\alpha_{i}\left(y_{i}\right)}=s_{i}
$$


then, we have

$$
\begin{aligned}
w\left(y_{i}\right) & \rightarrow w\left(Y_{i}\left(y_{i}\right)\right) \\
v_{j}\left(y_{i}\right) & \rightarrow v_{j}\left(Y_{i}\left(y_{i}\right)\right), \\
v_{i}\left(y_{i}\right) & \rightarrow Y_{i}^{\prime}\left(y_{i}\right) v_{i}\left(Y_{i}\left(y_{i}\right)\right), \\
q_{i}\left(y_{i}\right) & \rightarrow q_{i}\left(Y_{i}\left(y_{i}\right)\right)
\end{aligned}
$$

which in terms of the potential $W$ reads

$$
W\left(y_{i}\right) \rightarrow W\left(Y_{i}\left(y_{i}\right)\right)
$$

If we put $M_{0}=1$; i.e, we not consider the $t$-flow, the transformation is

$$
\begin{aligned}
& v_{i} \rightarrow X^{\prime}(x) v_{i}(X(x)), \\
& q_{i} \rightarrow X^{\prime}(x) q_{i}(X(x))+t_{00} \frac{X^{\prime \prime}}{X^{\prime}}
\end{aligned}
$$

where

$$
\int_{x}^{X} \frac{\mathrm{d} x}{\alpha_{0}(x)}=s_{0}
$$

That in potential form is

$$
W \rightarrow X^{\prime}(x) W(X(x))+\frac{t_{00}^{2}}{2} \frac{X^{\prime \prime}}{X^{\prime}} .
$$

This symmetry together with the $S_{i}$ symmetries described above constitute the well-known conformal symmetries of the extended Boyer-Finley system. When the $t$-flow is plugged in, and the extended Benney system appears, then this $x$-conformal symmetry disappears.

Nevertheless these additional symmetries, to the knowledge of the authors are not known for the generalized Benney system [32]

$$
\begin{aligned}
\frac{\partial q}{\partial t} & =\frac{\partial\left(q^{2}-2 w\right)}{\partial x} \\
\frac{\partial v}{\partial t} & =2 \frac{\partial(q v)}{\partial x} \\
\frac{\partial w}{\partial y} & =\frac{\partial v}{\partial x}
\end{aligned}
$$

In fact, we have proven

Proposition 6. Given any three functions $Y(y), f(t), T(t)$ and a solution $(w, q, v)$ of (92), then we have a new solution $(\tilde{w}, \tilde{q}, \tilde{v})$ given by

$$
\begin{aligned}
& \tilde{w}=T^{\prime}(t) w\left(\sqrt{T^{\prime}(t)}(x+f(t)), Y(y), T(t)\right)-\frac{t_{00}}{4} \frac{T^{\prime \prime}(t)}{T^{\prime}(t)}+\frac{1}{16}\{T, t\}_{S}(x+f(t))^{2}-\frac{f^{\prime \prime}(t)}{4}\left(x+\frac{f(t)}{2}\right), \\
& \tilde{q}=\sqrt{T^{\prime}(t)} q\left(\sqrt{T^{\prime}(t)}(x+f(t)), Y(y), T(t)\right)+\frac{1}{4} \frac{T^{\prime \prime}(t)}{T^{\prime}(t)}(x+f(t))-\frac{f^{\prime}(t)}{2}, \\
& \tilde{v}=\sqrt{T^{\prime}(t)} Y^{\prime}(y) v\left(\sqrt{T^{\prime}(t)}(x+f(t)), Y(y), T(t)\right) .
\end{aligned}
$$

We must notice that the above functional symmetries do not respect the shallow water reduction that appears in the limit $x=-y$. 


\subsection{Additional symmetries of Virasoro type and its action on string equations}

As we have seen in $§ 5.1$ additional symmetries appears when deformations of the initial con-

ditions are considered. Here we will consider initial conditions as in (73) and (71) with $G_{\mu}^{(0)}$ depending on a $s$ parameter as follows:

$$
G_{\mu}^{(0)}=\frac{x}{\hat{\xi}_{\mu}^{\prime}(p, s)}
$$

so that in the string equations (78) we will have functions

$$
f_{\mu}=f_{\mu}(s) .
$$

Notice that (93) describes a curve in the Virasoro algebra vir, and therefore describes the more general motion for the set of initial conditions $G_{\mu}$.

The right logarithmic derivative of the initial conditions (73) with respect to the additional parameter $s$ is

$$
\delta \mathrm{e}^{\operatorname{ad} G_{\mu}}\left(\frac{\partial}{\partial s}\right)=\beta_{\mu}\left(p_{\mu}\right) x_{\mu},, \quad \beta_{\mu}:=-\frac{f_{s}\left(p_{\mu}, s\right)}{f_{p_{\mu}}\left(p_{\mu}, s\right)},
$$

and the corresponding additional symmetry generator is

$$
F_{\mu}=\beta_{\mu}\left(z_{\mu}\right) m_{\mu}
$$

so that

$$
\frac{\partial z_{\nu}}{\partial s}=\beta_{\nu}\left(z_{\nu}\right)+\sum_{\mu=0}^{M}\left\{F_{(\mu,+)}, z_{\nu}\right\} .
$$

Now, if we freeze times $t_{\mu n}=0$ for $n>N_{\mu}$ so that

$$
m_{\mu}=\sum_{n=1}^{N_{\mu}} n t_{\mu n} z_{\mu}^{n-1}+t_{\mu 0} z_{\mu}^{-1}+\sum_{n=2}^{\infty} v_{\mu n} z_{\mu}^{-n}
$$

and we require the additional symmetry to leave those times invariant, we must have

$$
\beta_{\mu}\left(z_{\mu}\right)=\sum_{l=-1}^{\infty} b_{\mu l} z_{\mu}^{-l} .
$$

Let us take, for simplicity, Virasoro type generators

$$
\beta_{\nu}=c_{\nu} z_{\nu}^{1-n_{\nu}}, \quad n_{\nu}=1, \ldots, N_{\nu}, \quad c_{\nu} \in \mathbb{C}
$$

so that

$$
\frac{\partial z_{\nu}}{\partial s}=c_{\nu} z_{\nu}^{1-n_{\mu}}+\sum_{\substack{\mu=0, \ldots, M \\ n=1, \ldots, N_{\mu}-n_{\mu}}}\left(n+n_{\mu}\right) t_{\mu n+n_{\mu}} c_{\mu} \frac{\partial z_{\nu}}{\partial t_{\mu n}} .
$$

whose integration, leads to

$$
z_{\nu}(s)=\sqrt[n_{\mu}]{c_{\nu} n_{\nu} s+z_{\nu}(\boldsymbol{t}(s))^{n_{\mu}}} .
$$


where

$$
\begin{aligned}
& t_{\mu 1}(s):=t_{\mu 1}+\left(n_{\mu}+1\right) s c_{\mu} t_{\mu n_{\mu}+1}, \vdots \\
& \vdots \\
& t_{\mu N_{\mu}-n_{\mu}}(s):=t_{\mu N_{\mu}-n_{\mu}}+N_{\mu} s c_{\mu} t_{\mu N_{\mu}}, \\
& t_{\mu N_{\mu}-n_{\mu}+j}(s):=t_{\mu N_{\mu}-n_{\mu}+j, \quad j \geq 1 .}
\end{aligned}
$$

Integrating

$$
\frac{\partial f_{\mu}}{\partial s}+\beta_{\mu}\left(z_{\mu}\right) \frac{\partial f_{\mu}}{\partial z_{\mu}}=0
$$

we get

$$
f_{\mu}\left(z_{\mu}, s\right)=f_{\mu}\left(\sqrt[n_{\mu}]{-c_{\mu} n_{\mu} s+z_{\mu}^{n_{\mu}}(s)}\right)=f_{\mu}\left(z_{\mu}(\boldsymbol{t}(s))\right)
$$

\subsection{Invariance conditions for additional symmetries and string equations}

We note from (81) that the invariance condition under an additional symmetry

$$
F_{\mu}^{-}=\sum_{\nu=0}^{M}\left(F_{\nu}\right)_{(\nu,+)}-F_{\mu}=0, \quad \forall \mu,
$$

Thus, all the functions $F_{\mu}$ must reduce to a unique function $F_{\mu}=F \in \mathfrak{r}$. Given a solution of the string equations (70) we may take

$$
F_{\mu}=P_{\mu}^{1+r} Q_{\mu}^{1+s}
$$

and conclude, that $F_{\mu}=F \in \mathfrak{r}, \forall \mu$. Hence, string equations determine solutions invariant under additional symmetries characterized by the generators

$$
V_{\mu, r s}=P_{\mu}^{1+r} Q_{\mu}^{1+s}
$$

which close a Poisson algebra

$$
\left\{V_{\mu, r s}, V_{\mu, r^{\prime} s^{\prime}}\right\}=\left((r+1)\left(s^{\prime}+1\right)-\left(r^{\prime}+1\right)(s+1)\right) V_{r+r^{\prime}} s+s^{\prime} .
$$

In particular the functions $V_{r 0}$ generate a Virasoro algebra.

\section{A Appendix: The right logarithmic derivative}

Here we follow [9]. Given a manifold $\mathcal{T}$, a Lie group $G$ with Lie algebra $\mathfrak{g}$ and a map $\psi: \mathcal{T} \rightarrow G$ we define the right logarithmic derivative $\delta f \in \Omega^{1}(\mathcal{T}, \mathfrak{g})$ as the following $\mathfrak{g}$-valued 1-form

$$
\delta \psi(\xi)=T_{\psi(\boldsymbol{t})}\left(\mu^{\psi(\boldsymbol{t})^{-1}}\right) \circ T_{\boldsymbol{t}} \psi(\xi) \quad \forall \xi \in T_{\boldsymbol{t}} \mathcal{T}, \boldsymbol{t} \in \mathcal{T},
$$

where $\mu^{g}(h)=g \cdot h$ is the left multiplication in the Lie group. Recall that the right MaurerCartan form $\kappa \in \Omega^{1}(G, \mathfrak{g})$ is a $\mathfrak{g}$-valued 1-form over $G$ given by

$$
\kappa_{g}:=T_{g}\left(\mu^{g^{-1}}\right)
$$


in terms of which

$$
\delta \psi=\psi^{*} \kappa
$$

Given two maps $\psi, \phi: \mathcal{T} \rightarrow G$ then

$$
\delta(\psi \cdot \phi)=\delta \psi+\operatorname{Ad} \psi(\delta \phi)
$$

and therefore

$$
\delta\left(\psi^{-1}\right)=-\operatorname{Ad} \psi(\delta \psi) .
$$

It also holds for $\omega:=\delta \psi$ and $z=\operatorname{Ad} \psi(Z)$ that

$$
\begin{array}{r}
\mathrm{d} \omega+\frac{1}{2}[\omega, \omega]=0, \\
\mathrm{~d} z=[\delta \psi, z]+\operatorname{Ad} \psi(\mathrm{d} Z) .
\end{array}
$$

If there is an exponential mapping $\exp : \mathfrak{g} \rightarrow G$ we have the formula

$$
T_{X} \exp (Y)=T_{e} \mu^{\exp X} \cdot \int_{0}^{1} \operatorname{Ad}(\exp (s X)) Y \mathrm{~d} s .
$$

Thus, if $\psi=\exp X$ with $X: \mathcal{T} \rightarrow \mathfrak{g}$ we have

$$
\begin{aligned}
\delta \psi(\xi) & =T_{\psi} \mu^{\psi^{-1}}\left(T_{X} \exp \right) T_{\boldsymbol{t}} X(\xi)=T_{\psi} \mu^{\psi^{-1}} \circ T_{e} \mu^{\psi} \cdot \int_{0}^{1} \operatorname{Ad}(\exp (s X))\left(T_{\boldsymbol{t}} X(\xi)\right) \mathrm{d} s \\
& =\int_{0}^{1} \operatorname{Ad}(\exp (s X))\left(T_{\boldsymbol{t}} X(\xi)\right) \mathrm{d} s, \forall \xi \in T_{\boldsymbol{t}} \mathcal{T},
\end{aligned}
$$

that when we are allow to write $\operatorname{Ad} \exp X=\sum_{n=0}^{\infty}(\operatorname{ad} X)^{n} / n !-$ for example if $G$ is a Banach-Lie group- reads

$$
\delta \psi=\sum_{n=0}^{\infty} \frac{(\operatorname{ad} X)^{n} T_{\boldsymbol{t}} X}{(n+1) !} .
$$

Given a smooth curve $X: \mathbb{R} \rightarrow \mathfrak{g}$ we consider the problem

$$
\begin{aligned}
\delta \psi\left(\partial_{t}\right) & =X(t), \quad \psi: \mathbb{R} \rightarrow G \\
\psi(0) & =e .
\end{aligned}
$$

If there exists a solution is unique an local existence implies global existence. We write evol : $C^{\infty}(\mathbb{R}, \mathfrak{g}) \rightarrow G$, with $\operatorname{evol}(X(t))=g(1)$ and say, following Milnor, that the Lie group is regular is evol exists and is smooth. That is smooth curves in the Lie algebra integrates, in terms of the right logarithmic derivative, to smooth curves in the Lie group.

\section{Acknowledgements}

Partial economical support from Dirección General de Enseñanza Superior e Investigación Científica no BFM2002-01607, from European Science Foundation: MISGAM and from Marie Curie FP6 RTN ENIGMA is acknowledge. 


\section{References}

[1] O. Agam, E. Bettelheim, P. Wiegmann and A. Zabrodin, Phys. Rev. Lett. 88 (2002) 236801 .

[2] S. Aoyama and Y. Kodama, Commun. Math. Phys. 182, (1996) 185.

[3] A. Boyarsky, A. Marsahakov, O. Ruchaysky, P. Wiegmann and A. Zabrodin, Phys. Lett. B 515 (2001) 483.

[4] C. P. Boyer and J. D. Finley, J. Math. Phys. 23 (1982) 1126.

[5] Y. Gibbons and S.P. Tsarev, Phys. Lett A 258, (1999) 263

[6] F. Guil, M. Mañas and L. Martínez Alonso, J. Phys. A: Math. \& Gen. 36 (2003) 4047.

[7] F. Guil, M. Mañas and L. Martínez Alonso, J. Phys. A: Math.\& Gen. 36 (2003) 6457.

[8] V. Kazakov and A. Marsahakov, J. Phys. A: Math. \& Gen. 36 (2003) 3107.

[9] A. Kriegel, P. W. Michor, The Convenient Setting of Global Analysis, Mathematical Surveys and Monographs 53, American Mathematical Society (1997).

[10] B. Konopelchenko, L. Martinez Alonso and O. Ragnisco, A : Math. \& Gen. 34, (2001) 10209.

[11] I. M. Krichever, Func. Anal. Appl. 22 (1989) 200.

[12] I. M. Krichever, Comm. Pure Appl. Math. 47 (1992) 437.

[13] I. Krichever, M. Mineev-Weinstein, P. Wiegmann and A. Zabrodin, Physica D 198 (2004) 1.

[14] B. A. Kuperschmidt and Yu. I. Manin, Funk. Anal. Appl. 11, (3) ,31 (1977); 17, (1), 25 (1978).

[15] M. Mañas, The Principal Chiral Model as an Integrable System in Harmonic Maps and Integrable Systems, A. P. Fordy \& J. C. Wood (editors), Aspects of Mathematics 23 (1994) 147, Vieweg, Wiesbaden.

[16] M. Mañas, J. Phys. A: Math. Gen. 37 (2004) 9195.

[17] L. Martinez Alonso and M. Mañas, J. Math. Phys. 44 (2003) 3294.

[18] L. Martinez Alonso and E. Medina, J. Phys. A: Math. \& Gen. 37 (2004) 12005.

[19] L. Martinez Alonso and E. Medina, Phys. Lett. B 610 (2005) 227.

[20] L. Martinez Alonso, E. Medina and M. Mañas, String equations in the Whitham hierarchy: solutions and $\tau$-functions, to appear.

[21] M. Mineev-Weinstein, P. Wiegmann and A. Zabrodin, Phys. Rev. Lett. 84 (2000) 5106.

[22] A. G. Reiman and M. A. Semenov-Tyan-Shanskii, J. Sov. Math 46 (1986) 1631.

[23] K. Takasaki, Commun. Math. Phys. 170 (1995) 101.

[24] K. Takasaki and T. Takebe, Lett. Math. Phys. 23 (1991) 205.

[25] K. Takasaki and T. Takebe, Int. J. Mod. Phys. A7 Suppl.1 (1992) 889.

[26] K. Takasaki and T. Takebe, Lett. Math. Phys. 28 (1993) 165.

[27] K. Takasaki and T. Takebe, Rev. Math. Phys. 7 (1995) 743. 
[28] R. Teodorescu, E. Bettelheim, O. Agam, A. Zabrodin and P. Wiegmann, Nuc. Phys. B 700 (2004) 521; Nuc. Phys. B 704 (2005) 407.

[29] P. W. Wiegmann and P. B. Zabrodin, Comm. Math. Phys. 213 (2000) 523.

[30] A. Zabrodin, Teor. Mat. Fiz. 142 (2005) 197.

[31] V. E. Zakharov, Func. Anal. Priloz. 14, 89-98 (1980); Physica 3D, 193-202 (1981).

[32] V. E. Zakharov, Dispersionless limit of integrable systems in $2+1$ dimensions, in Singular Limits of Dispersive Waves, N.M. Ercolani et al. (editors), Plenum Press, NY, (1994) 165-174. 